\title{
Precise predictions for the Higgs-boson masses in the NMSSM
}

\author{
P. Drechsel ${ }^{1, \mathrm{a}}$, L. Galeta ${ }^{2, \mathrm{~b}}$, S. Heinemeyer ${ }^{2,3, \mathrm{c}}$, G. Weiglein ${ }^{1, \mathrm{~d}}$ \\ ${ }^{1}$ DESY, Notkestraß e 85, 22607 Hamburg, Germany \\ ${ }^{2}$ Instituto de Física de Cantabria (CSIC-UC), Santander, Spain \\ ${ }^{3}$ Instituto de Física Teórica, (UAM/CSIC), Universidad Autónoma de Madrid, Cantoblanco, 28049 Madrid, Spain
}

Received: 18 August 2016 / Accepted: 1 January 2017 / Published online: 21 January 2017

(C) The Author(s) 2017. This article is published with open access at Springerlink.com

\begin{abstract}
The particle discovered in the Higgs-boson searches at the LHC with a mass of about $125 \mathrm{GeV}$ can be identified with one of the neutral Higgs bosons of the Nextto-Minimal Supersymmetric Standard Model (NMSSM). We calculate predictions for the Higgs-boson masses in the NMSSM using the Feynman-diagrammatic approach. The predictions are based on the full NMSSM one-loop corrections supplemented with the dominant and sub-dominant two-loop corrections within the Minimal Supersymmetric Standard Model (MSSM). These include contributions at $\mathcal{O}\left(\alpha_{t} \alpha_{s}, \alpha_{b} \alpha_{s}, \alpha_{t}^{2}, \alpha_{t} \alpha_{b}\right)$, as well as a resummation of leading and subleading logarithms from the top/scalar top sector. Taking these corrections into account in the prediction for the mass of the Higgs boson in the NMSSM that is identified with the observed signal is crucial in order to reach a precision at a similar level as in the MSSM. The quality of the approximation made at the two-loop level is analysed on the basis of the full one-loop result, with a particular focus on the prediction for the Standard Model-like Higgs boson that is associated with the observed signal. The obtained results will be used as a basis for the extension of the code FeynHiggs to the NMSSM.
\end{abstract}

\section{Introduction}

The spectacular discovery of a boson with a mass around $125 \mathrm{GeV}$ by the ATLAS and CMS experiments [1,2] at CERN constitutes a milestone in the quest for understanding the physics of electroweak symmetry breaking. Any model describing electroweak physics needs to provide a state that can be identified with the observed signal. While within

\footnotetext{
a e-mail: peter.drechsel@desy.de

be-mail: leo@ifca.unican.es

c e-mail: Sven.Heinemeyer@cern.ch

de-mail: Georg.Weiglein@desy.de
}

the present experimental uncertainties the properties of the observed state are compatible with the predictions of the Standard Model (SM) [3,4], many other interpretations are possible as well, in particular as a Higgs boson of an extended Higgs sector.

One of the prime candidates for physics beyond the SM is supersymmetry (SUSY), which doubles the particle degrees of freedom by predicting two scalar partners for all SM fermions, as well as fermionic partners to all bosons. The most widely studied SUSY framework is the Minimal Supersymmetric Standard Model (MSSM) [5,6], which keeps the number of new fields and couplings to a minimum. In contrast to the single Higgs doublet of the (minimal) SM, the Higgs sector of the MSSM contains two Higgs doublets, which in the $\mathcal{C P}$ conserving case leads to a physical spectrum consisting of two $\mathcal{C P}$-even, one $\mathcal{C P}$-odd and two charged Higgs bosons. The light $\mathcal{C} \mathcal{P}$-even MSSM Higgs boson can be interpreted as the signal discovered at about $125 \mathrm{GeV}$; see e.g. $[7,8]$.

Going beyond the MSSM, this model has a well-motivated extension in the Next-to-Minimal Supersymmetric Standard Model (NMSSM); see e.g. [9,10] for reviews. The NMSSM provides in particular a solution for naturally associating an adequate scale to the $\mu$ parameter appearing in the MSSM superpotential $[11,12]$. In the NMSSM, the introduction of a new singlet superfield, which only couples to the Higgs and sfermion sectors, gives rise to an effective $\mu$-term, generated in a similar way as the Yukawa mass terms of fermions through its vacuum expectation value. In the case where $\mathcal{C P}$ is conserved, which we assume throughout the paper, the states in the NMSSM Higgs sector can be classified as three $\mathcal{C P}$-even Higgs bosons, $h_{i}(i=1,2,3)$, two $\mathcal{C P}$-odd Higgs bosons, $A_{j}(j=1,2)$, and the charged Higgs-boson pair $H^{ \pm}$. In addition, the SUSY partner of the singlet Higgs (called the singlino) extends the neutralino sector to a total of five neutralinos. In the NMSSM the lightest but also the sec- 
ond lightest $\mathcal{C P}$-even neutral Higgs boson can be interpreted as the signal observed at about $125 \mathrm{GeV}$; see, e.g., $[13,14]$.

The measured mass value of the observed signal has already reached the level of a precision observable, with an experimental accuracy of better than $300 \mathrm{MeV}$ [15], and by itself provides an important test for the predictions of models of electroweak symmetry breaking. In the MSSM the masses of the $\mathcal{C P}$-even Higgs bosons can be predicted at lowest order in terms of two SUSY parameters characterising the MSSM Higgs sector, e.g. $\tan \beta$, the ratio of the vacuum expectation values of the two doublets, and the mass of the $\mathcal{C P}$-odd Higgs boson, $M_{A}$, or the charged Higgs boson, $M_{H^{ \pm}}$. These relations, which in particular give rise to an upper bound on the mass of the light $\mathcal{C P}$-even Higgs boson given by the $Z$-boson mass, receive large corrections from higher-order contributions. In the NMSSM the corresponding predictions are modified both at the tree level and the loop level. In order to fully exploit the precision of the experimental mass value for constraining the available parameter space of the considered models, the theoretical predictions should have an accuracy that ideally is at the same level of accuracy or even better than the one of the experimental value. The theoretical uncertainty, on the other hand, is composed of two sources, the parametric and the intrinsic uncertainty. The theoretical uncertainties induced by the parametric errors of the input parameters are dominated by the experimental error of the top-quark mass (where the latter needs to include the systematic uncertainty from relating the measured mass parameter to a theoretically well-defined quantity; see e.g. [16-18]). However, the largest theoretical uncertainty at present arises from unknown higher-order corrections, as will be discussed below.

In the $\operatorname{MSSM}^{1}$ beyond the one-loop level, the dominant two-loop corrections of $\mathcal{O}\left(\alpha_{t} \alpha_{s}\right)$ [19-24] and $\mathcal{O}\left(\alpha_{t}^{2}\right)$ [25,26] as well as the corresponding corrections of $\mathcal{O}\left(\alpha_{b} \alpha_{s}\right)$ [27,28] and $\mathcal{O}\left(\alpha_{t} \alpha_{b}\right)$ [27] are known since more than a decade. (Here we use $\alpha_{f}=Y_{f}^{2} /(4 \pi)$, with $Y_{f}$ denoting the fermion Yukawa coupling.) These corrections, together with a resummation of leading and subleading logarithms from the top/scalar top sector [29] (see also [30,31] for more details on this type of approach), a resummation of leading contributions from the bottom/scalar bottom sector [27, 28,32-35] (see also [36,37]) and momentum-dependent two-loop contributions [38,39] (see also [40]) are included in the public code FeynHiggs [21,29,41-45]. A (nearly) full two-loop EP calculation, including even the leading three-loop corrections, has also been published [46,47], which is, however, not publicly available as a computer code. Furthermore, another leading three-loop calculation of $\mathcal{O}\left(\alpha_{t} \alpha_{s}^{2}\right)$, depending on the various SUSY mass hierarchies, has been performed $[48,49]$,

\footnotetext{
1 As mentioned above, we focus in this paper on the case of real parameters, i.e. the $\mathcal{C} \mathcal{P}$-conserving case.
}

resulting in the code $\mathrm{H} 3 \mathrm{~m}$ (which adds the three-loop corrections to the FeynHiggs result up to the two-loop level). The theoretical uncertainty on the lightest $\mathcal{C P}$-even Higgsboson mass within the MSSM from unknown higher-order contributions is still at the level of about $3 \mathrm{GeV}$ for scalar top masses at the $\mathrm{TeV}$-scale, where the actual uncertainty depends on the considered parameter region [29,43,50,51].

Within the NMSSM beyond the well-known full oneloop results [52-55] several codes exist that calculate the Higgs masses in the pure $\overline{\mathrm{DR}}$ scheme with different contributions at the two-loop level. Amongst these codes SPheno [56,57] incorporates the most complete results at the two-loop level, including SUSY-QCD contributions from the fermion/sfermions of $\mathcal{O}\left(\alpha_{t} \alpha_{s}, \alpha_{b} \alpha_{s}\right)$, as well as pure fermion/sfermion contributions of $\mathcal{O}\left(\alpha_{t}^{2}, \alpha_{b}^{2}, \alpha_{t} \alpha_{b}, \alpha_{\tau}^{2}\right.$, $\alpha_{\tau} \alpha_{b}$ ), and contributions from the Higgs/higgsino sector in the gauge-less limit of $\mathcal{O}\left(\alpha_{\lambda}^{2}, \alpha_{\kappa}^{2}, \alpha_{\lambda} \alpha_{\kappa}\right)$ [58] as well as mixed contributions from the latter two sectors of $\mathcal{O}\left(\alpha_{\lambda} \alpha_{t}, \alpha_{\lambda} \alpha_{b}\right)$. The included Higgs/higgsino contributions are genuine to the NMSSM, they are proportional to the NMSSM parameters $\lambda^{2}=4 \pi \cdot \alpha_{\lambda}$ and $\kappa^{2}=4 \pi \cdot \alpha_{\kappa}$. The tools FlexiblesUSY [59], NMSSMTools [60,61] and SOFTSUSY [62-64] include NMSSM corrections of $\mathcal{O}\left(\alpha_{t} \alpha_{s}\right)$ and $\mathcal{O}\left(\alpha_{b} \alpha_{s}\right)$ supplemented by certain MSSM corrections. NMSSMCalc $[54,55,65,66]$ provides the option to perform the NMSSM Higgs-mass calculation up to $\mathcal{O}\left(\alpha_{t} \alpha_{s}\right)$ with the $\overline{\mathrm{DR}}$ renormalisation scheme applied to the top/stop sector, while in the electroweak sector at one-loop order onshell conditions are used. It has been noticed in a comparison of spectrum generators in the NMSSM that are currently publicly available that the numerical differences between the various codes can be very significant, often exceeding $3 \mathrm{GeV}$ in the prediction of the SM-like Higgs even for the set-up where all predictions were obtained within the $\overline{\mathrm{DR}}$ renormalisation scheme [67]. While the sources of discrepancies between the different codes could be identified [67], a reliable estimate of the remaining theoretical uncertainties should of course also address issues related to the use of different renormalisation schemes. Beyond the pure $\overline{\mathrm{DR}}$ scheme, so far only the code NMSSMCalc $[54,55,65,66]$ provides a prediction in a mixed OS/DR scheme, where genuine two-loop contributions in the NMSSM up to $\mathcal{O}\left(\alpha_{t} \alpha_{s}\right)$ have been incorporated. The resummation of logarithmic contributions beyond the two-loop level is not included so far in any of the public codes for Higgs-mass predictions in the NMSSM. Accordingly, at present the theoretical uncertainties from unknown higher-order corrections in the NMSSM are expected to be still larger than for the MSSM.

Concerning the phenomenology of the NMSSM it is of particular interest whether this model can be distinguished from the MSSM by confronting Higgs-sector measurements with the corresponding predictions of the two models. In order to facilitate the identification of genuine NMSSM con- 
tributions in this context it is important to treat the predictions for the MSSM and the NMSSM within a coherent framework where in the MSSM limit of the NMSSM the state-of-the-art prediction for the MSSM is recovered.

With this goal in mind, we seek to extend the public tool FeynHiggs to the case of the NMSSM. As a first step in this direction we present in this paper a full one-loop calculation of the Higgs-boson masses in the NMSSM, where the renormalisation scheme and all parameters and conventions are chosen such that the well-known MSSM result of FeynHiggs is obtained for the MSSM limit of the NMSSM. We supplement the full one-loop result in the NMSSM with all higher-order corrections of MSSM-type that are implemented in FeynHiggs, as described above. In our numerical evaluation we use our full one-loop result in the NMSSM to assess the quality of the approximation that we make at the two-loop level. We find that for a SM-like Higgs boson that is compatible with the detected signal at about $125 \mathrm{GeV}$ this approximation works indeed very well. We analyse in this context which genuine NMSSM contributions are most relevant when going beyond the approximation based on MSSMtype higher-order corrections. We then apply our most accurate prediction including all higher-order contributions to four phenomenologically interesting scenarios. We compare our prediction both with the result in the MSSM limit and with the code NMSSMCalc [65]. We discuss in this context the impact of higher-order contributions beyond the ones of $\mathcal{O}\left(\alpha_{t} \alpha_{s}\right)$, which are not implemented in NMSSMCalc.

The paper is organised as follows. In Sect. 2 we describe our full one-loop calculation in the NMSSM, specify the renormalisation scheme that we have used and discuss the contributions that are expected to be numerically dominant at the one-loop level. The incorporation of higher-order contributions of MSSM-type is addressed in Sect. 3. Our numerical analysis for the prediction at the one-loop level, including a discussion of the quality of the approximation in terms of MSSM-type contributions, and for our most accurate prediction including higher-order corrections is presented in Sect. 4. The conclusions can be found in Sect. 5.

\section{One-loop result in the NMSSM}

For the sectors that are identical for the calculation within the MSSM the conventions as implemented in FeynHiggs are used, as described in [44]. Therefore the present section is restricted to the quantities genuine to the NMSSM. For a more detailed discussion of the NMSSM; see e.g. [9].

\subsection{The relevant NMSSM sectors}

The superpotential of the NMSSM for the third generation of fermions/sfermions reads

$$
\begin{aligned}
W= & Y_{t}\left(\hat{H}_{2} \cdot \hat{Q}_{3}\right) \hat{u}_{3}-Y_{d}\left(\hat{H}_{1} \cdot \hat{Q}_{3}\right) \hat{d}_{3} \\
& -Y_{\tau}\left(\hat{H}_{1} \cdot \hat{L}_{3}\right) \hat{e}_{3}+\lambda \hat{S}\left(\hat{H}_{2} \cdot \hat{H}_{1}\right)+\frac{1}{3} \kappa \hat{S}^{3},
\end{aligned}
$$

with the quark and lepton superfields $\hat{Q}_{3}, \hat{u}_{3}, \hat{d}_{3}, \hat{L}_{3}, \hat{e}_{3}$ and the Higgs superfields $\hat{H}_{1}, \hat{H}_{S}, \hat{S}$. The $S U(2)_{\mathrm{L}}$-invariant product is denoted by a dot. The Higgs singlet and doublets are decomposed into $\mathcal{C P}$-even and $\mathcal{C P}$-odd neutral scalars $\phi_{i}$ and $\chi_{i}$, and charged states $\phi_{i}^{ \pm}$,

$H_{1}=\left(\begin{array}{c}v_{1}+\frac{1}{\sqrt{2}}\left(\phi_{1}-i \chi_{1}\right) \\ -\phi_{1}^{-}\end{array}\right)$,

$H_{2}=\left(\begin{array}{c}\phi_{2}^{+} \\ v_{2}+\frac{1}{\sqrt{2}}\left(\phi_{2}+i \chi_{2}\right)\end{array}\right)$,

$S=v_{s}+\frac{1}{\sqrt{2}}\left(\phi_{s}+i \chi_{s}\right)$,

with the real vacuum expectation values for the doublet- and the singlet fields, $v_{\{1,2\}}$ and $v_{s}$. Since $\hat{S}$ transforms as a singlet, the $D$-terms remain identical to the ones from the MSSM. Compared to the $\mathcal{C P}$-conserving MSSM the superpotential of the $\mathcal{C P}$-conserving NMSSM contains additional dimensionless parameters $\lambda$ and $\kappa$, while the $\mu$-term is absent. This term is effectively generated via the vacuum expectation value of the singlet field,

$\mu_{\mathrm{eff}}=\lambda v_{s}$

As in the MSSM it is convenient to define the ratio

$\tan \beta=\frac{v_{2}}{v_{1}}$.

Soft SUSY-breaking in the NMSSM gives rise to the real trilinear soft-breaking parameters $A_{\lambda}$ and $A_{\kappa}$, as well as to the soft-breaking mass term $m_{S}^{2}$ of the scalar singlet field,

$$
\begin{aligned}
\mathcal{L}_{\mathrm{soft}}= & -m_{1}^{2} H_{1 i}^{\dagger} H_{1 i}-m_{2}^{2} H_{2 i}^{\dagger} H_{2 i}-m_{S}^{2}|S|^{2} \\
& -\left[\lambda A_{\lambda} S\left(H_{2} \cdot H_{1}\right)+\frac{1}{3} \kappa A_{\kappa} S^{3} .+ \text { h.c. }\right] .
\end{aligned}
$$

The Higgs potential $V_{H}$ can be written in powers of the fields,

$$
\begin{aligned}
V_{\mathrm{H}}= & \cdots-T_{\phi_{1}} \phi_{1}-T_{\phi_{2}} \phi_{2}-T_{\phi_{S}} \phi_{s} \\
& +\frac{1}{2}\left(\phi_{1}, \phi_{2}, \phi_{s}\right) \mathbf{M}_{\phi \phi}\left(\begin{array}{c}
\phi_{1} \\
\phi_{2} \\
\phi_{s}
\end{array}\right) \\
& +\frac{1}{2}\left(\chi_{1}, \chi_{2}, \chi_{s}\right) \mathbf{M}_{\chi \chi}\left(\begin{array}{c}
\chi_{1} \\
\chi_{2} \\
\chi_{s}
\end{array}\right) \\
& +\left(\phi_{1}^{-}, \phi_{2}^{-}\right) \mathbf{M}_{\phi^{ \pm} \phi^{ \pm}}\left(\begin{array}{c}
\phi_{1}^{+} \\
\phi_{2}^{+}
\end{array}\right)+\cdots,
\end{aligned}
$$


where the coefficients bilinear in the fields are the mass matrices $\mathbf{M}_{\phi \phi}, \mathbf{M}_{\chi \chi}$ and $\mathbf{M}_{\phi^{ \pm} \phi^{ \pm}}$. For the $\mathcal{C} \mathcal{P}$-even fields the (symmetric) mass matrix reads
Also in Eq. (11) $\mu_{\text {eff }}$ can have a significant influence on the mixing between the singlino and the doublet higgsino fields. For instance, for sufficiently large values of $\mu_{\mathrm{eff}}$ such that

$\mathbf{M}_{\phi \phi}=\left(\begin{array}{ccc}\hat{m}_{A}^{2} s_{\beta}^{2}-M_{Z}^{2} c_{\beta}^{2}\left(\hat{m}_{A}^{2}+M_{Z}^{2}\right) s_{\beta} c_{\beta} & \mu_{\mathrm{eff}}\left(2 \lambda v c_{\beta}-\kappa v s_{\beta}\right)+\frac{\lambda v}{\mu_{\mathrm{eff}}} c_{\beta} \\ \cdot & \hat{m}_{A}^{2} c_{\beta}^{2}-M_{Z}^{2} s_{\beta}^{2} & \mu_{\mathrm{eff}}\left(2 \lambda v s_{\beta}-\kappa v c_{\beta}\right)+\frac{\lambda v}{\mu_{\mathrm{eff}}} s_{\beta} \\ \cdot & \cdot & \lambda \kappa v^{2} c_{\beta} s_{\beta}+\frac{\lambda^{2} v^{2}}{\mu_{\mathrm{eff}}^{2}} \hat{m}_{A}^{2}+\frac{\kappa}{\lambda} \mu_{\mathrm{eff}}\left(4 \frac{\kappa}{\lambda} \mu_{\mathrm{eff}}+A_{\kappa}\right)\end{array}\right)$,

where $s_{\beta}$ and $c_{\beta}$ denote the sine and cosine of the angle $\beta$, and

$\hat{m}_{A}^{2}=M_{H^{ \pm}}^{2}-M_{W}^{2}+\lambda^{2} v^{2}$.

The lower triangle in Eq. (7) is filled with the transposed matrix element. For the $\mathcal{C P}$-conserving case the mixing into the eigenstates of mass and $\mathcal{C P}$ can be described at lowest order by the following unitary transformations:

$$
\begin{aligned}
\left(\begin{array}{l}
h_{1} \\
h_{2} \\
h_{3}
\end{array}\right) & =\mathbf{U}_{e(0)}\left(\begin{array}{l}
\phi_{1} \\
\phi_{2} \\
\phi_{s}
\end{array}\right), \quad\left(\begin{array}{l}
A_{1} \\
A_{2} \\
G_{0}
\end{array}\right)=\mathbf{U}_{o(0)}\left(\begin{array}{l}
\chi_{1} \\
\chi_{2} \\
\chi_{s}
\end{array}\right), \\
\left(\begin{array}{l}
H^{ \pm} \\
G^{ \pm}
\end{array}\right) & =\mathbf{U}_{c(0)}\left(\begin{array}{l}
\phi_{1}^{ \pm} \\
\phi_{2}^{ \pm}
\end{array}\right) .
\end{aligned}
$$

The matrices $\mathbf{U}_{\{e, o, c\}(0)}$ transform the Higgs fields such that the mass matrices are diagonalised at tree level. The new fields correspond to the five neutral Higgs bosons $h_{i}$ and $A_{j}$, the charged pair $H^{ \pm}$, and the Goldstone bosons $G^{0}$ and $G^{ \pm}$.

In Eq. (7) the third row and column depend explicitly on $\mu_{\text {eff. }}$ The numerical value of $\mu_{\text {eff }}$ has an important impact on the singlet admixture after performing the rotation into the mass eigenstate basis. For instance, for values of $\mu_{\text {eff }}$ large enough so that

$\left(\mathbf{M}_{\phi \phi}\right)_{33} \gg\left(\mathbf{M}_{\phi \phi}\right)_{i 3}, \quad i \in\{1,2\}$,

the mass of the singlet becomes decoupled from the doublet masses.

The superpartner of the scalar singlet appears as a fifth neutralino. The corresponding $5 \times 5$ mass matrix reads

$\mathbf{Y}=\left(\begin{array}{ccccc}M_{1} & 0 & -M_{Z} s_{\mathrm{W}} \cos \beta & M_{Z} s_{\mathrm{W}} \sin \beta & 0 \\ 0 & M_{2} & M_{Z} c_{\mathrm{W}} \cos \beta & -M_{Z} c_{\mathrm{w}} \sin \beta & 0 \\ -M_{Z} s_{\mathrm{W}} \cos \beta & M_{Z} c_{\mathrm{W}} \cos \beta & 0 & -\mu_{\mathrm{eff}} & \lambda v \sin \beta \\ M_{Z} s_{\mathrm{W}} \sin \beta & -M_{Z} c_{\mathrm{W}} \sin \beta & -\mu_{\mathrm{eff}} & 0 & \lambda v \cos \beta \\ 0 & 0 & \lambda v \sin \beta & \lambda v \cos \beta & -2 \frac{\kappa}{\lambda} \mu_{\mathrm{eff}}\end{array}\right)$.

It is diagonalised by a unitary matrix

$\mathbf{D}_{\mathbf{Y}}=\mathbf{N}^{*} \mathbf{Y} \mathbf{N}^{\dagger}=\operatorname{diag}\left\{m_{\tilde{\chi}_{1}^{0}}, m_{\tilde{\chi}_{2}^{0}}, m_{\tilde{\chi}_{3}^{0}}, m_{\tilde{\chi}_{4}^{0}}, m_{\tilde{\chi}_{5}^{0}}\right\}$.
$(\mathbf{Y})_{55} \gg(\mathbf{Y})_{i 5}, \quad i \in\{3,4\}$,

the singlino mass decouples from the masses of higgsinos and gauginos.

\subsection{Renormalisation scheme}

In order to derive the counterterms entering the one-loop corrections to the Higgs-boson masses the independent parameters appearing in the linear and bilinear terms of the Higgs potential in Eq. (6) have to be renormalised. The set of independent parameters from the Higgs sector used for the presented calculation is formed by

MSSM-like: $\quad T_{h_{1,2}}, \mu_{\mathrm{eff}}, M_{H^{ \pm}}^{2}, \tan \beta, M_{W}^{2}, M_{Z}^{2}$

genuine NMSSM: $T_{h_{3}}, \kappa, \lambda, A_{\kappa}, v=\sqrt{v_{1}^{2}+v_{2}^{2}}$.

Here $T_{h_{i}}$ denotes the tadpole coefficient for the field $h_{i}$ (as indicated by the subscript) in the mass eigenstate basis. The relation to the tadpoles in the interaction basis, $T_{\phi_{i}}$, is given by

$$
\left(\begin{array}{l}
T_{h_{1}} \\
T_{h_{2}} \\
T_{h_{3}}
\end{array}\right)=\mathbf{U}_{e(0)}\left(\begin{array}{c}
T_{\phi_{1}} \\
T_{\phi_{2}} \\
T_{\phi_{s}}
\end{array}\right)
$$

The soft-breaking mass terms are related to the tadpole coefficients by

$$
\begin{aligned}
m_{1}= & -\frac{T_{\phi_{1}}}{\sqrt{2} v \sin \beta}-\mu_{\mathrm{eff}}^{2}+\mu_{\mathrm{eff}} B \tan \beta \\
& -\lambda^{2} v^{2} \sin ^{2} \beta+\frac{1}{4} M_{Z}^{2}\left(\sin ^{2} \beta-\cos ^{2} \beta\right) \\
m_{2}= & -\frac{T_{\phi_{2}}}{\sqrt{2} v \cos \beta}-\mu_{\mathrm{eff}}^{2}+\mu_{\mathrm{eff}} B \cot \beta \\
& -\lambda^{2} v^{2} \cos ^{2} \beta+\frac{1}{4} M_{Z}^{2}\left(\cos ^{2} \beta-\sin ^{2} \beta\right) \\
m_{s}^{2}= & -\frac{\lambda T_{\phi_{s}}}{\sqrt{2} \mu_{\mathrm{eff}}}+\left(\mu_{\mathrm{eff}} B \frac{\lambda^{2} v^{2}}{\mu_{\mathrm{eff}}^{2}}+\lambda \kappa v^{2}\right) \\
& \times \sin \beta \cos \beta-\lambda^{2} v^{2}-2 \frac{\kappa^{2}}{\lambda^{2}} \mu_{\mathrm{eff}}^{2}+\frac{\kappa}{\lambda} \mu_{\mathrm{eff}} A_{\kappa},
\end{aligned}
$$


where

$$
\begin{aligned}
\mu_{\mathrm{eff}} B= & \frac{1}{\sqrt{2} v}\left(\sin ^{3} \beta T_{\phi_{1}}+\cos ^{3} \beta T_{\phi_{2}}\right) \\
& +\left(M_{H^{ \pm}}^{2}-M_{W}^{2}+\lambda^{2} v^{2}\right) \sin \beta \cos \beta
\end{aligned}
$$

and

$\mu_{\mathrm{eff}} B=\mu_{\mathrm{eff}}\left(\frac{\kappa}{\lambda} \mu_{\mathrm{eff}}+A_{\lambda}\right)$.

Using these equations the original soft-breaking mass parameters $m_{1}, m_{2}$ and $m_{s}^{2}$ are replaced by $T_{h_{1}, h_{2}, h_{3}}$, and the softbreaking trilinear parameter $A_{\lambda}$ is replaced by $M_{H^{ \pm}}$.

Parameters that do not enter the MSSM calculation are considered as genuine of the calculation in the NMSSM. Although the vacuum expectation value $v$ is not a parameter genuine to the NMSSM, its appearance as an independent parameter is a specific feature of the NMSSM Higgs-mass calculation; see below.

For all parameters appearing only in the NMSSMcalculation, besides the additional tadpole coefficient, a $\overline{\mathrm{DR}}$ scheme is applied. This is a difference from the calculations performed in $[54,55,65,66]$, where the electric charge $e$ is renormalised instead of the parameter $v$. These two parameters are related to each other by

$$
\begin{aligned}
v & =\frac{\sqrt{2} s_{\mathrm{W}} M_{W}}{e} \rightarrow v\left(1+\frac{\delta v}{v}\right) \\
& =v\left[1+\frac{1}{2}\left(\frac{\delta M_{W}^{2}}{M_{W}^{2}}+\frac{\delta s_{\mathrm{W}}^{2}}{s_{\mathrm{w}}^{2}}-2 \delta Z_{e}\right)\right],
\end{aligned}
$$

with the renormalisation constants for the $W$-boson mass, $\delta M_{W}^{2}$, the sine of the Weinberg angle, $\delta s_{\mathrm{w}}^{2}$, where $s_{\mathrm{w}}^{2} \equiv$ $1-M_{W}^{2} / M_{Z}^{2}, s_{\mathrm{w}}^{2}+c_{\mathrm{w}}^{2}=1$, and the electric charge is renormalised as

$e \rightarrow e\left(1+\delta Z_{e}\right)$.

Considering $\delta M_{W}^{2}$ and $\delta s_{\mathrm{w}}^{2}$ already fixed by on-shell conditions for the gauge-boson masses [44], either $\delta Z_{e}$ or $\delta v$ in Eq. (19) can be fixed by an independent renormalisation condition (and the other counterterm is then a dependent quantity). The renormalisation prescription [54] where $\delta Z_{e}$ is fixed by renormalising $e$ in the static limit results in a non$\overline{\mathrm{DR}}$ renormalisation for $\delta v$. For the self-energies in the Higgs sector $\delta v$ enters the counterterms for the renormalised Higgs potential,

$V_{\mathrm{H}} \rightarrow V_{\mathrm{H}}+\delta V_{\mathrm{H}}$,

with coefficients involving $\lambda$ and $\kappa$, like

$$
\left.\frac{\delta^{(2)}}{\delta \phi_{s} \delta \phi_{i}} \delta V_{\mathrm{H}}\right|_{\phi_{l}, \chi_{m}, \phi_{n}^{ \pm}=0} \supset-\kappa \mu_{\mathrm{eff}}\{\sin \beta, \cos \beta\}(\delta v+\cdots),
$$

for the self-energies with each an external doublet and singlet field. The ellipses in Eq. (22) denote other renormalisation constants that are fixed in the $\overline{\mathrm{DR}}$-scheme and thus do not contribute with a finite part. However, a finite contribution from $\delta v$ would lead to a $\kappa$-dependence of all loop contributions entering via $\delta v$, in particular also of the corrections from the fermions and sfermions (while the fermion and sfermion contributions to the unrenormalised self-energy are $\kappa$-independent). A finite contribution from $\delta v$ would furthermore imply the rather artificial feature that a self-energy involving an external gauge singlet field would receive a counterterm contribution involving the renormalisation constant $\delta Z_{e}$ for the electric charge. We therefore prefer to use the $\overline{\mathrm{DR}}$-scheme for the renormalisation of $v$, which means that we use a scheme where $\delta Z_{e}$ is a dependent counterterm. This leads to the relation

$\delta Z_{e}^{\mathrm{dep}}=\frac{1}{2}\left[\frac{\delta s_{\mathrm{w}}^{2}}{s_{\mathrm{w}}^{2}}+\frac{\delta M_{W}^{2}}{M_{W}^{2}}-\frac{\delta v^{2}}{v^{2}}\right]$

which implies

$\left.\delta Z_{e}^{\mathrm{dep}}\right|^{\text {fin }}=\frac{1}{2}\left[\frac{\delta s_{\mathrm{w}}^{2}}{s_{\mathrm{w}}^{2}}+\frac{\delta M_{W}^{2}}{M_{W}^{2}}\right]^{\text {fin }}$

for the finite part of $\delta Z_{e}^{\text {dep }}$. In this scheme the numerical value for the electric charge $e$ (and accordingly for the electromagnetic coupling constant $\alpha$ ) is determined indirectly via Eq. (24). In order to avoid a non-standard numerical value for $\alpha$ in our numerical results, we apply a two-step procedure: in the first step we apply a $\overline{\mathrm{DR}}$ renormalisation for $v$ as outlined above. As a second step we then reparametrise this result in terms of a suitably chosen expression for $\alpha$. By default we use the same convention as for the MSSM result that is implemented in FeynHiggs, namely the expression for the electric charge in terms of the Fermi constant $G_{F}$, in order to facilitate the comparison between the FeynHiggs result in the MSSM and our new result in the NMSSM. Taking the MSSM limit of our new NMSSM result, the MSSM result as implemented in FeynHiggs is recovered, since the described calculational differences are genuine NMSSM effects that vanish in this limit. For the numerical comparison with NMSSMCalc we will use instead $\alpha\left(M_{Z}\right)$. The procedure of the reparametrisation is outlined in the following section. 


\subsection{Reparametrisation of the electromagnetic coupling}

The couplings $g^{\mathrm{I}}$ and $g^{\mathrm{II}}$ in two different renormalisation schemes are in general related to each other by

$g^{\mathrm{I}}\left(1+\delta Z_{g}^{\mathrm{I}}\right)=g^{\mathrm{II}}\left(1+\delta Z_{g}^{\mathrm{II}}\right)$

because of the equality of the bare couplings. The corresponding shift in the numerical values of the coupling definitions is obtained from the finite difference of the two counterterms, $\Delta \equiv g^{\mathrm{II}} \delta Z_{g}^{\mathrm{II}}-g^{\mathrm{I}} \delta Z_{g}^{\mathrm{I}}$. Accordingly, a reparametrisation from the numerical value of the coupling used in scheme I to the one of scheme II can be performed via

$g^{\mathrm{I}}=g^{\mathrm{II}}+\Delta$.

Since $\Delta$ is of one-loop order, its insertion into a tree-level expression generates a term of one-loop order, etc.

In our calculation the reparametrisation of the electromagnetic coupling is only necessary up to the one-loop level, since all corrections of two-loop and higher order that we are going to incorporate have been obtained in the gaugeless limit (some care is necessary regarding the incorporation of the MSSM-type contributions of $\mathcal{O}\left(\alpha_{t}^{2}\right)$; see $\left.[26,68]\right)$. At this order the shift $\Delta$ can simply be expressed as $\Delta=$ $g^{\mathrm{II}}\left(\delta Z_{g}^{\mathrm{II}}-\delta Z_{g}^{\mathrm{I}}\right)$. Specifically, for the reparametrisation of the electromagnetic coupling constant $G_{F}$ the parameter shift $\Delta_{G_{F}}$ reads

$\Delta_{G_{F}}=e\left(\delta Z_{e}-\delta Z_{e}^{\mathrm{dep}}-\frac{1}{2} \Delta r^{\mathrm{NMSSM}}\right)$.

Here $\delta Z_{e}$ is the counterterm of the charge renormalisation within the NMSSM according to the static (Thomson) limit,

$\delta Z_{e}=\frac{1}{2} \Pi^{\gamma \gamma}(0)+\frac{s_{\mathrm{w}}}{c_{\mathrm{w}}} \frac{\Sigma_{T}^{\gamma Z}(0)}{M_{Z}^{2}}$,

and $\Pi^{\gamma \gamma}(0), \Sigma_{T}^{\gamma Z}(0)$ are the derivative of the transverse part of the photon self-energy and the transverse part of the photon- $Z$ self-energy at zero momentum transfer, respectively. The counterterm $\delta Z_{e}^{\text {dep }}$ has been defined in Eq. (23), and for the quantity $\Delta r^{\mathrm{NMSSM}}$ we use the result of [69] (see also [70]). ${ }^{2}$ The numerical value for the electromagnetic coupling $e$ in this parametrisation is obtained from the Fermi constant in the usual way as $e=2 M_{W} s_{\mathrm{w}} \sqrt{G_{F} \sqrt{2}}$.

Similarly, for the reparametrisation of the electromagnetic coupling defined in the previous section in terms of $\alpha\left(M_{Z}\right)$ the parameter shift $\Delta_{\alpha\left(M_{Z}\right)}$ reads

${ }^{2}$ For the sample scenario defined in Table 2 below the numerical value of $\Delta r^{\text {NMSSM }}$ from [69] turns out to be close to $3.8 \%$, with only a weak dependence on $\lambda$ for the range of $\lambda$ values discussed in this paper.
$\Delta_{\alpha\left(M_{Z}\right)}=e\left(\delta Z_{e}-\delta Z_{e}^{\mathrm{dep}}-\frac{1}{2} \Delta \alpha\right)$

The numerical value of $e$ in this parametrisation is obtained from $\alpha\left(M_{Z}\right)=\alpha(0) /(1-\Delta \alpha)$, and $\alpha(0)$ is the value of the fine-structure constant in the Thomson limit.

\subsection{Dominant contributions at one-loop order}

As explained above, we will supplement our full one-loop result with all available higher-order contributions of MSSMtype. This means in particular that the two-loop contributions are approximated by the two-loop corrections in the MSSM (i.e. omitting genuine NMSSM corrections) as included in FeynHiggs, and further corrections beyond the two-loop level are included. In order to validate this approximation we analyse at the one-loop level the size of genuine NMSSM corrections w.r.t. the MSSM-like contributions.

Since the corrections from the top/stop sector are usually the by far dominant ones, we start with a qualitative discussion of those contributions before we perform a numerical analysis in the following section. In the MSSM the leading corrections from the top/stop sector are commonly denoted as $\mathcal{O}\left(\alpha_{t}\right)$, indicating the occurrence of two Yukawa couplings $Y_{t}$. In the limit where all other masses of the SM particles and the external momentum are neglected compared to the top-quark mass, for dimensional reasons the correction to the squared Higgs-boson mass furthermore receives a contribution proportional to $m_{t}^{2}$. This gives rise to the well-known coefficient $G_{F} m_{t}^{4}$ of the leading one-loop contributions. In the NMSSM the formally leading contributions either are of $\mathcal{O}\left(Y_{t}^{2}\right)$ (involving two Yukawa couplings), of $\mathcal{O}\left(\lambda Y_{t}\right)$ (involving one Yukawa coupling), or of $\mathcal{O}\left(\lambda^{2}\right)$ (involving no Yukawa coupling). The various contributions from the top/stop sector are summarised in Table 1. The contributions in the second column are the ones of MSSM-type, while the entries in the third through fifth column represent the genuine NMSSM corrections, involving only scalar tops. ${ }^{3}$

For the doublet fields, the couplings between the Higgsand stop-fields in the gauge-less limit are proportional to the top-quark Yukawa coupling,

$i \Gamma_{\phi_{2} \tilde{t}_{i} \tilde{t}_{j}}=i \sqrt{2} Y_{t}\left[A_{t} \cdot c_{i j}^{\phi_{2}}\left(\theta_{\tilde{t}}\right)+m_{t} \cdot(-1)^{1-i} \delta_{i j}\right]$,
$i \Gamma_{\phi_{1} \tilde{t}_{i} \tilde{t}_{j}}=i \sqrt{2} Y_{t} \mu_{\mathrm{eff}} \cdot c_{i j}^{\phi_{1}}\left(\theta_{\tilde{t}}\right)$,

while the corresponding coupling for the singlet field reads

$i \Gamma_{\phi_{s} \tilde{t}_{i} \tilde{t}_{j}}=i \sqrt{2} \lambda \cot \beta m_{t} \cdot c_{i j}^{\phi_{s}}\left(\theta_{\tilde{t}}\right)$.

\footnotetext{
3 We discuss here only the Higgs-boson self-energies. However, the same line of argument can be made for the tadpole contributions.
} 
Table 1 Topologies and their order in terms of the couplings in the top/stop sector that contribute to the self-energies of the $\mathcal{C} \mathcal{P}$-even fields $\phi_{i}$ at one-loop order in the gauge-less limit. The numbers 1 and 2 denote the doublet-states as external fields, while $s$ denotes an external singlet. The internal lines depict either a top (solid) or a scalar top (dashed)

\begin{tabular}{lllll}
\hline$(i, j)$ & $(1|2,1| 2)$ & $(1, s)$ & $(2, s)$ & $(s, s)$ \\
\hline Order & $\mathcal{O}\left(Y_{t}^{2}\right)$ & $\mathcal{O}\left(\lambda Y_{t}\right)$ & $\mathcal{O}\left(\lambda Y_{t}\right)$ & $\mathcal{O}\left(\lambda^{2}\right)$ \\
Fields & Top/stop & Stop & Stop & \\
Topologies & & &
\end{tabular}

The non-vanishing quartic Higgs-stop couplings read

$i \Gamma_{\phi_{2} \phi_{2} \tilde{t}_{i} \tilde{t}_{j}}=-i Y_{t}^{2} \cdot \delta_{i j}, \quad i \Gamma_{\phi_{1} \phi_{s} \tilde{t}_{i} \tilde{t}_{j}}=-i \lambda Y_{t} \cdot c_{i j}^{\phi_{1} \phi_{s}}\left(\theta_{\tilde{t}}\right)$.

Here functions of the mixing angle of the stop sector, $\theta_{\tilde{t}}$, are denoted by $c$ with the appropriate indices and superscripts for the involved fields. These functions $c$ can never be larger than 1. In the singlet-stop coupling we have explicitly spelled out a factor $\lambda v_{1} Y_{t}=\lambda m_{t} \cot \beta$ to highlight the appearance of the factor $m_{t}$ in Eq. (30b) instead of the usual factor $m_{t} / M_{W} \sim$ $Y_{t}$ in Eq. (30a).

The genuine NMSSM couplings of a singlet to stops are seen to follow the pattern mentioned above, i.e. they give rise to contributions of $\mathcal{O}\left(\lambda Y_{t}\right)$ (third and fourth column in Table 1) or $\mathcal{O}\left(\lambda^{2}\right)$ (fifth column), whereas the MSSM-like contributions are of $\mathcal{O}\left(Y_{t}^{2}\right)$ (second column). Those different patterns do not only indicate a distinction between the MSSM-like and the genuine NMSSM contributions, but also give rise to a significant numerical suppression ${ }^{4}$ of the genuine NMSSM contributions w.r.t. the MSSM-like ones for $\lambda<Y_{t}$. If one demands validity of perturbation theory up to the GUT scale, this relation is always fulfilled, since then $\lambda$ and $\kappa$ are bound from above [12] by

$\lambda^{2}+\kappa^{2} \lesssim 0.5$

so that $\lambda \lesssim 0.7$, where the largest values are only allowed for vanishing $\kappa$. The size of the genuine NMSSM contributions will be discussed numerically in the following sections.

\section{Incorporation of higher-order contributions}

The masses of the $\mathcal{C P}$-even Higgs bosons are obtained from the complex poles of the full propagator matrix. The inverse

\footnotetext{
${ }^{4}$ For the trilinear couplings in Eqs. 30a, 30b, comparing the Higgs singlet with the doublet, an additional potential suppression factor of $\mathcal{O}\left(m_{t} / A_{t}\right)$ and/or $\mathcal{O}\left(m_{t} / \mu_{\text {eff }}\right)$ appears.
}

propagator matrix for the three $\mathcal{C P}$-even Higgs bosons $h_{i}$ from Eq. (9) is a $3 \times 3$ matrix that reads

$\Delta^{-1}\left(k^{2}\right)=i\left[k^{2} \mathbb{1}-\mathcal{M}_{h h}+\hat{\Sigma}_{h h}\left(k^{2}\right)\right]$.

Here $\mathcal{M}_{h h}$ denotes the diagonalised mass matrix of the $\mathcal{C P}$ even Higgs fields at tree level, and $\hat{\Sigma}_{h h}$ denotes their renormalised self-energy corrections. ${ }^{5}$ The three complex poles of the propagator in the $\mathcal{C P}$-even Higgs sector are given by the values of the external momentum $k$ for which the determinant of the inverse propagator-matrix vanishes,

$\operatorname{det}\left[\Delta^{-1}\left(k^{2}\right)\right]_{k^{2}=m_{h_{i}}^{2}-i \Gamma_{h_{i}} m_{h_{i}}} \stackrel{!}{=} 0, \quad i \in\{1,2,3\}$.

The real parts of the three poles are identified with the square of the Higgs-boson masses in the $\mathcal{C P}$-even sector. The renormalised self-energy matrix $\hat{\Sigma}_{h h}$ is evaluated by taking into account the full contributions from the NMSSM at one-loop order and, as an approximation, the MSSM-like contributions at two-loop order of $\mathcal{O}\left(\alpha_{t} \alpha_{s}, \alpha_{b} \alpha_{s}, \alpha_{t}^{2}, \alpha_{t} \alpha_{b}\right)$ at vanishing external momentum taken over from FeynHiggs [21,29,41-45], where also the resummation of leading and subleading logarithms from the top/scalar top sector is incorporated [29], ${ }^{6}$

$\left.\hat{\Sigma}_{h h}\left(k^{2}\right) \approx \hat{\Sigma}_{h h}^{(1 \mathrm{~L})}\left(k^{2}\right)\right|^{\mathrm{NMSSM}}+\left.\hat{\Sigma}_{h h}^{(2 \mathrm{~L}+\text { beyond })}\left(k^{2}\right)\right|_{k^{2}=0} ^{\mathrm{MSSM}}$

In order to facilitate the incorporation of the MSSM-like twoloop contributions from FeynHiggs, the renormalisation scheme chosen for the NMSSM contributions closely follows the FeynHiggs conventions as described in [44]. Accordingly, the stop masses are renormalised on-shell. For our

\footnotetext{
5 Details on the calculation of the renormalised self-energy contributions will be presented in a future publication.

${ }^{6}$ In the public version of FeynHiggs for the NMSSM also the recent results for momentum-dependent two-loop contributions in the MSSM of $[38,39]$ will be implemented.
} 
Table 2 Definition of the sample scenario, S. All dimensionful parameters are given in GeV. All $\overline{\mathrm{DR}}$-parameters are defined at $m_{t}^{\overline{\mathrm{MS}}}\left(m_{t}\right)$. All stop-parameters are on-shell parameters. As indicated by the super- script "(GUT)", $M_{1}$ is related to $M_{2}$ by the usual GUT relation, $M_{1}^{(\mathrm{GUT})}=5 s_{\mathrm{w}}^{2} /\left(3 c_{\mathrm{w}}^{2}\right) M_{2}$

\begin{tabular}{|c|c|c|c|c|c|c|c|c|}
\hline \multicolumn{5}{|c|}{ Higgs-sector parameters } & \multicolumn{4}{|c|}{ Heavy fermion masses } \\
\hline$M_{H^{ \pm}}$ & $\tan \beta$ & $\mu_{\mathrm{eff}}$ & $A_{\kappa}$ & $\kappa$ & $m_{t}^{\mathrm{OS}}$ & $m_{t}^{\overline{\mathrm{MS}}}\left(m_{t}\right)$ & $m_{b}^{\overline{\mathrm{MS}}}\left(m_{b}\right)$ & $m_{\tau}$ \\
\hline 1000 & 8 & 125 & -300 & 0.2 & 173.2 & 167.48 & 4.2 & 1.78 \\
\hline
\end{tabular}

Sfermion and gaugino parameters

\begin{tabular}{|c|c|c|c|c|c|c|c|}
\hline$M_{\tilde{q}}$ & $M_{\tilde{l}}$ & $A_{t}$ & $A_{\tau}, A_{b}, A_{q}$ & $A_{l}$ & $M_{1}^{(\mathrm{GUT})}$ & $M_{2}$ & $M_{3}$ \\
\hline 1500 & 200 & -2000 & -1500 & -100 & $\approx 143$ & 300 & 1500 \\
\hline$M_{\tilde{q}}$ & \multicolumn{7}{|c|}{ Universal squark mass breaking parameter } \\
\hline$M_{\tilde{l}}$ & \multicolumn{7}{|c|}{ Universal slepton mass breaking parameter } \\
\hline$A_{t / b / q}$ & \multicolumn{7}{|c|}{ Trilinear breaking term for stop-/sbottom/the lighter squark generations } \\
\hline$A_{\tau / l}$ & \multicolumn{7}{|c|}{ Trilinear breaking term for stau/the two lighter slepton generations } \\
\hline$M_{\{1,2,3\}}$ & \multicolumn{7}{|c|}{ Gaugino mass breaking 1 parameters for $U(1)_{\mathrm{Y}}, S U(2)_{\mathrm{L}}, S U(3)_{\mathrm{c}}$} \\
\hline
\end{tabular}

numerical evaluation below we employ the MSSM contributions obtained from the version FeynHiggs $2.10 .2 .^{7}$ The poles of the inverse propagator matrix are determined numerically. The algorithm for this procedure is the same as the one described in [54]. For the generation and calculation of the self-energies the tools FeynArts 3.9 [71,72] and FormCalc $7.4[73,74]$ have been used. The implementation of the NMSSM with real parameters was based on a FeynArts model file generated by SARAH [75-78].

\section{Numerical results}

A particular goal of our numerical analysis is to test the kind of approximation in terms of MSSM-type contributions that we have used at the two-loop level. For this purpose a genuine NMSSM scenario will be studied, which gives rise to a SM-like Higgs with a predicted mass at the two-loop level of around $125 \mathrm{GeV}$ and a singlet-like Higgs field with a mass that can be above or below the one of the SM-like state. In order to investigate the influence of the extended Higgs and higgsino sector of the NMSSM compared to the MSSM the parameter $\lambda$ will be varied. In the limit $\lambda \rightarrow 0$ and constant $\mu_{\text {eff }}$ all singlet fields decouple from the remaining field spectrum. Increasing the value of $\lambda$ directly translates to increasing the influence of genuine NMSSM-effects. A detailed study of the one-loop result and the quality of approximations based on partial contributions will be presented here. In order to study the approximation of making the restriction of MSSM-like contributions beyond oneloop order at $\mathcal{O}\left(\alpha_{t} \alpha_{s}\right)$, we will compare our result with the

\footnotetext{
$\overline{7}$ More recent updates of FeynHiggs contain additional contributions that, however, do not significantly modify the results of our present investigation.
}

public tool NMSSMCalc [65], which incorporates the genuine NMSSM-type contributions of $\mathcal{O}\left(\alpha_{t} \alpha_{s}\right)$ using a hybrid $\overline{\mathrm{DR}}$ /on-shell renormalisation scheme. While for the MSSM various other higher-order corrections are implemented in FeynHiggs, the corresponding contributions have not been taken into account in NMSSMCalc. We will compare in this context the numerical effect of the NMSSM-type contributions of $\mathcal{O}\left(\alpha_{t} \alpha_{s}\right)$ as implemented in NMSSMCalc with the MSSM-type contributions of this order, and we will investigate the numerical impact of the MSSM-type corrections beyond $\mathcal{O}\left(\alpha_{t} \alpha_{s}\right)$.

In our numerical discussion below we will just focus on the masses of the two lighter $\mathcal{C P}$-even states. The effects discussed below turn out to be very small for the heaviest $\mathcal{C P}$-even state, amounting to less than $1 \%$ for the considered scenarios.

\subsection{Numerical scenarios and treatment of input parameters}

In our study we will discuss four different scenarios. The first "sample scenario", S, for our study is defined by the parameters given in Table 2. It has been chosen to exemplify typical features of NMSSM phenomenology and is well suited for studying the magnitude of the NMSSM-contributions and the behaviour of the employed approximation. The second and third scenario are the benchmark scenarios P1 and P9 defined in [79], where the parameter $\lambda$ is varied. While the original motivation for these scenarios arising from the diphoton excess that was observed by ATLAS [80] and CMS [81] in the 2015 Run 2 data has not received support from the latest data, we use those scenarios here to serve as examples of possible NMSSM phenomenology in order to test to what extent the features visible for the "sample scenario" S also apply to completely different scenarios. The fourth scenario 
Table 3 Definition of the analysed scenarios P1, P9 and A1. All dimensionful parameters are given in $\mathrm{GeV}$. All $\overline{\mathrm{DR}}$-parameters are considered to be defined at $m_{t}^{\overline{\mathrm{MS}}}\left(m_{t}\right)$, and all stop-parameters are considered to be on-shell parameters. The remaining trilinear breaking parameters are chosen as $A_{f}=1500 \mathrm{GeV}$. The parameter $\hat{m}_{A}$ is related to the charged Higgs $M_{H^{ \pm}}$mass by Eq. (8)

\begin{tabular}{|c|c|c|c|c|c|c|c|c|c|c|c|}
\hline & \multicolumn{5}{|c|}{ Higgs-sector parameters } & \multicolumn{6}{|c|}{ Sfermion and gaugino parameters } \\
\hline & $\hat{m}_{A}$ & $\tan \beta$ & $\mu_{\text {eff }}$ & $A_{\kappa}$ & $\kappa$ & $M_{\tilde{q}}$ & $M_{\tilde{l}}$ & $A_{t}$ & $M_{1}$ & $M_{2}$ & $M_{3}$ \\
\hline P1 & 760 & 10 & 150 & 0 & 0.25 & 1750 & 300 & -4000 & 500 & 1000 & 3000 \\
\hline \multirow[t]{2}{*}{ P9 } & 765 & 14 & 110 & 0 & 0.17 & 2050 & 400 & -4000 & 500 & 1000 & 3000 \\
\hline & $M_{H^{ \pm}}$ & $\tan \beta$ & $\mu_{\text {eff }}$ & $A_{\kappa}$ & $\kappa$ & $M_{\tilde{q}}$ & $M_{\tilde{l}}$ & $A_{t}$ & $M_{1}$ & $M_{2}$ & $M_{3}$ \\
\hline A1 & 1500 & 10 & 150 & 0 & 0.25 & 1750 & 300 & -4000 & 500 & 1000 & 3000 \\
\hline
\end{tabular}

A1 is based on P1, but permits much larger values of $\lambda$. The Higgs-sector parameters of $\mathrm{P} 1, \mathrm{P} 9$ and $\mathrm{A} 1$ are given in Table 3. Throughout our analysis the parameter $\lambda$ is varied if not stated otherwise. We will show in our numerical discussion below that the qualitative features of the scenarios P1, P9 and A1 can be understood from the discussion of the "sample scenario".

The choice for the top-quark mass in the loop contributions will be the pole mass $m_{t}^{\mathrm{OS}}$ for the comparison with NMSSMCalc and $m_{t}^{\overline{\mathrm{MS}}}\left(m_{t}\right)$ for the remaining studies. Using the $\overline{\mathrm{MS}}$ top-quark mass allows us to include the resummation of leading and next-to-leading logarithms implemented in FeynHiggs. The renormalisation scale for the studies in this chapter will be fixed at the used value of the top-quark mass.

\subsubsection{Sample scenario $S$}

The sample scenario $S$ for our study is defined by the parameters given in Table 2 . For values $\lambda \gtrsim 0.32$ the mass of the lightest state becomes tachyonic at tree level for this scenario, and therefore the analyses will be performed only for values of $\lambda$ up to 0.32 .

The viability of the discussed scenario is tested with the full set of experimental data implemented in the tool HiggsBounds 4 .1 .3 [82-86]. In order to obtain the necessary input for HiggsBounds we made use of NMSSMTOOls 4.4.0 [9] and linked it with HiggsBounds. While our calculation assumes an on-shell renormalised stop sector as in [44], the SLHA input file for NMSSMTOols needs $\overline{\mathrm{DR}}$-parameters for the stop sector. Thus a conversion from the on-shell into the $\overline{\mathrm{DR}}$ scheme is necessary for the parameters of the sample scenario given in Table 2. We only accounted for the dominant effect of these conversions that occurs for $X_{t}=A_{t}-\mu_{\mathrm{eff}} \cot \beta$ by applying the on-shell to $\overline{\mathrm{DR}}$ conversion outlined in [87]. We find that the scenario is in agreement with the experimental limits implemented in HiggsBounds 4.1.3.

\subsubsection{Scenarios with $A_{\kappa}=0$ and very large $\left|A_{t}\right|$}

The scenarios $\mathrm{P} 1$ and $\mathrm{P} 9$ are defined by the parameters given in Table 3. They are characterised in particular by the choice of $A_{\kappa}=0$ and very large (negative) $A_{t}$. While in the original definition of [79] the values $\lambda=0.1$ and $\lambda=0.05$ were chosen for the scenarios $\mathrm{P} 1$ and $\mathrm{P} 9$, respectively, we vary the parameter $\lambda$ here. We nevertheless refer to the scenarios as $\mathrm{P} 1$ and $\mathrm{P} 9$ also for other values of $\lambda$ for simplicity.

In the scenario $\mathrm{P} 1$ for all values of $\lambda \gtrsim 0.43$ the lightest Higgs state becomes tachyonic, for scenario P9 this is the case for $\lambda \gtrsim 0.35$. The analyses will therefore be restricted to values of $\lambda \lesssim 0.43$ for the scenario $\mathrm{P} 1$ and $\lambda \lesssim 0.35$ for the scenario $\mathrm{P} 9$, respectively. The parameters entering at higher order are chosen as given in Table 3 in the same fashion as above.

\subsubsection{Example of a scenario with large values of $\lambda$}

The scenario $\mathrm{A} 1$ is based on $\mathrm{P} 1$, but with a substantially larger value of $M_{H^{ \pm}}$, which prevents tachyonic Higgs masses at the tree level even for large values of $\lambda$. The parameters are given in the lower part of Table 3. Although we found that this scenario is in disagreement with experimental data from the Tevatron and the LHC Run 1 for $\lambda \gtrsim 0.75$, it permits the analysis of the MSSM-approximation also for very large values of $\lambda$.

\subsection{Full results at two-loop order}

The full results for the tree level, one- and two-loop Higgsmass predictions in the discussed scenarios defined in Tables 2 and 3 are shown as a function of $\lambda$ in Fig. 1 for the two lighter $\mathcal{C} \mathcal{P}$-even fields. The term "full result" refers to all one-loop corrections in the NMSSM (including the full momentum dependence and also the reparametrisation of the electromagnetic coupling in terms of the Fermi constant), supplemented with all available contributions of $\mathcal{O}\left(\alpha_{t} \alpha_{s}, \alpha_{b} \alpha_{s}, \alpha_{t}^{2}, \alpha_{t} \alpha_{b}\right)$ from the MSSM, and including the resummation of large logarithms. 

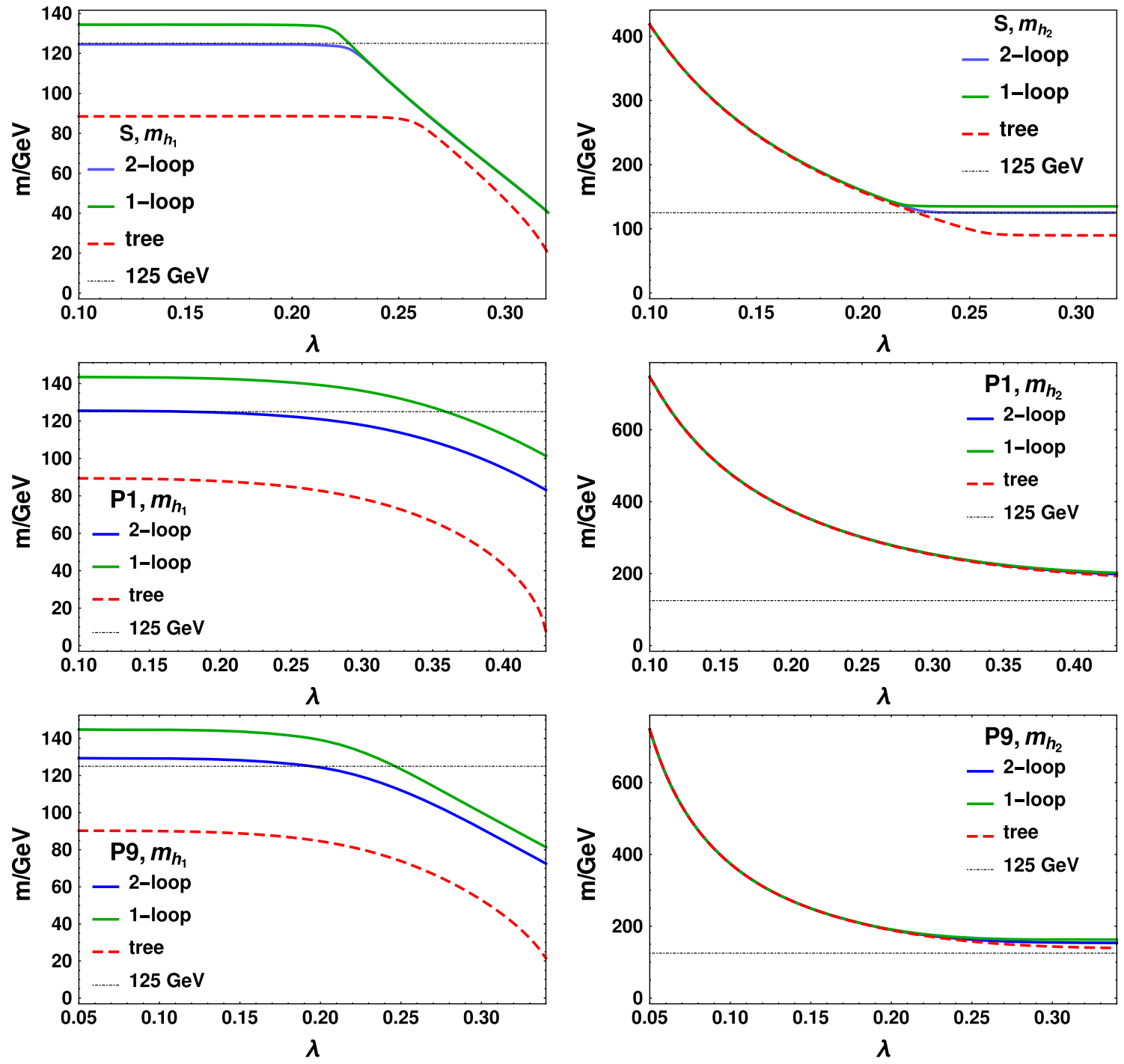

Fig. 1 Mass of the lightest and next-to lightest $\mathcal{C P}$-even Higgsstates, $m_{h_{1}}$ (left) and $m_{h_{2}}$ (right), at tree level, one-loop and twoloop order for the sample scenario (first row), the scenarios P1 (second row) and P9 (third row). At one-loop order all corrections of the NMSSM are included with their momentum dependence. The twoloop corrections are approximated by the MSSM-type contributions of $\mathcal{O}\left(\alpha_{t} \alpha_{s}, \alpha_{b} \alpha_{s}, \alpha_{t}^{2}, \alpha_{t} \alpha_{b}\right)$ including the resummation of the leading and next-to-leading logarithms (see text). The dotted line represents

\subsubsection{Sample scenario $S$}

The results for the sample scenario S, as defined in Table 2, are shown in the first row of Fig. 1. For this study the parameter $\lambda$ is varied between 0.1 and 0.32 . The lower limit on the parameter $\lambda$ has been chosen such that in the considered parameter region a cross-over type behaviour occurs only for the two smaller masses, $m_{h_{1}}$ and $m_{h_{2}}$ (for values $\lambda<0.1$ there is another point with cross-over behaviour of the two larger Higgs-boson masses; however, because of the small values of $\lambda$ this region is less suitable for studying the

$125 \mathrm{GeV}$. The $\lambda$ values for which a cross-over behaviour between the masses occurs in the sample scenario are at the tree-level $\lambda_{c}^{(0)} \approx 0.26$, at one-loop order $\lambda_{\mathrm{c}}^{(1)} \approx 0.22$ and at two-loop order $\lambda_{\mathrm{c}}^{(2)} \approx 0.23$. In the scenario $\mathrm{P} 9$ a cross-over behaviour occurs at $\lambda_{\mathrm{c}}^{(0)}>0.34$ at tree level, at $\lambda_{\mathrm{c}}^{(1)} \approx 0.25$ at one-loop order, and at $\lambda_{\mathrm{c}}^{(2)} \approx 0.26$ at two-loop order. In the scenario P1 the cross-over behaviour occurs outside of the plotted interval

behaviour of the genuine NMSSM-corrections, which scale with $\lambda$ ).

The variation of the two masses with $\lambda$ in the first row of Fig. 1 clearly shows a cross-over type behaviour between the masses, which is correlated to their mixing character w.r.t. the singlet field and the doublet fields. For small values of $\lambda$ the field $h_{1}$ is doublet-like in this scenario and, based on the prediction incorporating all available higher-order corrections, can be identified with the signal that was detected at the LHC at about $125 \mathrm{GeV}$. The prediction for $m_{h_{1}}$ varies only very little with $\lambda$ in this region. The field $h_{2}$, on the other hand, is predominantly singlet-like in this parameter 


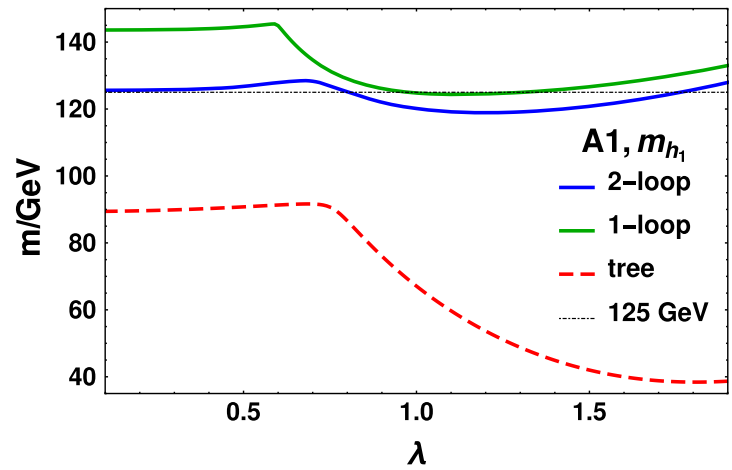

Fig. 2 Mass of the lightest and next-to lightest $\mathcal{C} \mathcal{P}$-even Higgs-states, $m_{h_{1}}$ (left), $m_{h_{2}}$ (right), two-loop order for the scenario A1. The included corrections are identical to the ones in Fig. 1 . The $\lambda$ values for which

region, and its mass prediction falls steeply with increasing $\lambda$. The cross-over occurs at $\lambda_{\mathrm{c}}^{(0)} \approx 0.26$ at tree level, at $\lambda_{\mathrm{c}}^{(1)} \approx 0.22$ at one-loop order, and at $\lambda_{\mathrm{c}}^{(2)} \approx 0.23$ at two-loop order. Above the cross-over point the behaviour of the two masses and the admixture of the fields $h_{1}$ and $h_{2}$ in terms of singlet and doublet fields are reversed. The two fields are evenly mixed between singlet- (i.e., genuine NMSSM-type) and doublet-field (i.e., MSSM-type) components for $\lambda_{\mathfrak{c}}^{(n)}$, with $n=0,1,2$. The heaviest $\mathcal{C P}$-even Higgs field, $h_{3}$, is doublet-like in the depicted interval of $\lambda$. As in the MSSM, the larger masses (of doublet-like fields) are affected by higher-order corrections to a lesser extent than the lighter states. Since at $\lambda_{c}^{(n)}$ the MSSM-type and genuine NMSSMtype contributions enter at equal footing, the SM-like state is most sensitive to genuine NMSSM-type contributions in the region of the cross-over behaviour.

\subsubsection{Scenario $P 1$}

The results for the scenario $\mathrm{P} 1$ are shown in the second row of Fig. 1. The lightest field is dominantly doublet-like, and the second lightest state is singlet-like for the depicted values of $\lambda$. The cross-over region between the doublet- and singletlike state is rather wide in this case and starts at $\lambda \approx 0.2$. The cross-over would occur for values of $\lambda$ above 0.43 , where the lightest field becomes tachyonic at the tree level (this parameter region is therefore not shown here). Thus, even for the largest value of $\lambda \approx 0.43$ shown in the plot the lightest field is still dominantly doublet-like at all depicted orders. We therefore find that the qualitative behaviour in this scenario is very similar to the sample scenario, but the allowed range of $\lambda$ is restricted to the region below the cross-over point in this case. For small values of $\lambda$ the lightest field can be identified with the signal that was detected at the LHC at about $125 \mathrm{GeV}$. The heaviest $\mathcal{C} \mathcal{P}$-even Higgs field (not shown

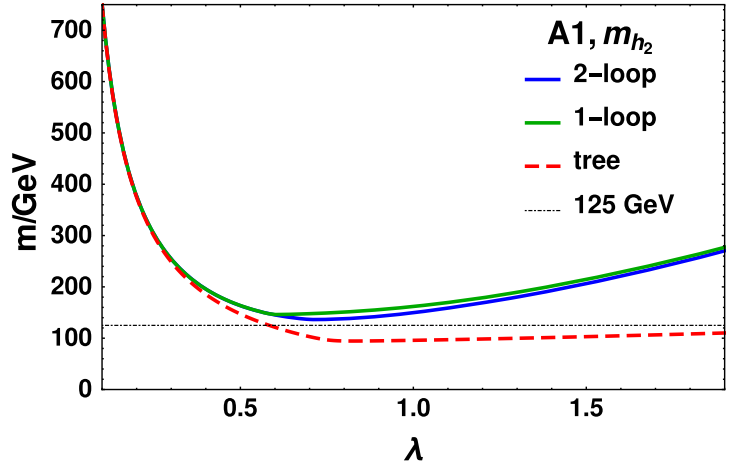

a cross-over behaviour between the masses occurs are at the tree-level $\lambda_{\mathrm{c}}^{(0)} \approx 0.75$, at one-loop order $\lambda_{\mathrm{c}}^{(1)} \approx 0.70$ and at two-loop order $\lambda_{\mathrm{c}}^{(2)} \approx 0.62$

in the figure) remains doublet-like with a nearly constant mass of $\approx 760 \mathrm{GeV}$ for the depicted values of $\lambda$.

\subsubsection{Scenario P9}

The results for the scenario P9 are shown in the third row of Fig. 1. Similarly to scenario P1 the variation of the two masses with $\lambda$ follows the behaviour of the sample scenario. The interval in which the cross-over behaviour occurs is larger than in the sample scenario, but smaller than in scenario P1. The cross-over occurs at $\lambda_{\mathrm{c}}^{(0)}>0.34$ at tree level, at $\lambda_{\mathrm{c}}^{(1)} \approx 0.25$ at one-loop order, and at $\lambda_{\mathrm{c}}^{(2)} \approx 0.26$ at two-loop order. It thus lies within the displayed $\lambda$ range if loop corrections are taken into account. While as before the character of the lightest field $h_{1}$ changes from dominantly doublet-like to dominantly singlet-like when $\lambda$ is increased through the cross-over region (and vice versa for $h_{2}$ ), $h_{1}$ retains a doublet admixture of more than $40 \%$ even for $\lambda$ values above the cross-over region in this scenario. Because of the sizeable admixture in this region, $m_{h_{1}}$ and $m_{h_{2}}$ each receive significant self-energy contributions from both the singlet and the doublet fields. The heaviest $\mathcal{C P}$-even Higgs field (not shown in the figure) remains doublet-like with a nearly constant mass of $\approx 750 \mathrm{GeV}$ for the depicted values of $\lambda$.

\subsubsection{Scenario Al}

The results for the scenario A1 are shown in Fig. 2. For values $\lambda \lesssim 0.75$ the variation of the masses with $\lambda$ follows the behaviour of the sample scenario. In this region the lightest field is doublet-like and the second lightest field is singletlike. The $\lambda$ values for which a cross-over behaviour occurs are at the tree-level $\lambda_{\mathrm{c}}^{(0)} \approx 0.75$, at one-loop order $\lambda_{\mathrm{c}}^{(1)} \approx 0.70$ and at two-loop order $\lambda_{\mathrm{c}}^{(2)} \approx 0.62$. For larger values of $\lambda$ the lightest field $h_{1}$ obtains a singlet admixture of roughly $70 \%$, and the next-to lightest field $h_{2}$ obtains a doublet admixture 
of the same size. A doublet-like Higgs field with a mass close to $125 \mathrm{GeV}$ can be realised only for values of $\lambda$ smaller than $\lambda_{\mathrm{c}}$ in this scenario. The heaviest $\mathcal{C} \mathcal{P}$-even Higgs field remains doublet-like with a mass increasing from nearly 1500 to $1580 \mathrm{GeV}$ for the displayed values of $\lambda$. In the following we will omit the discussion of the heaviest $\mathcal{C} \mathcal{P}$-even Higgs field, since it receives only very small two-loop contributions.

4.3 Numerically leading contributions at the one-loop level

For the prediction in the MSSM the top/stop-sector contributions are numerically leading. In the studied scenarios, given in Tables 2 and 3, the genuine NMSSM-corrections are suppressed w.r.t. the corresponding MSSM-like stop-corrections since $\lambda \lesssim \lambda_{\max }<Y_{t}$, where $\lambda_{\max }=0.32,0.43,0.35$ in the three scenarios, see the discussion in Sect. 2.4. Thus, the genuine NMSSM corrections from this sector are expected to be subleading.

In order to study the impact of the genuine NMSSM contributions we compare the approximation based on the leading MSSM-type one-loop corrections in the gauge-less limit of $\mathcal{O}\left(Y_{t}^{2}\right)$, labelled " $t / \tilde{t}$-MSSM" in Fig. 3, with the one where the genuine NMSSM corrections of $\mathcal{O}\left(\lambda Y_{t}, \lambda^{2}\right)$ are incorporated.

\subsubsection{Sample scenario}

For the sample scenario the difference between the mass predictions in the two approximations is plotted as a function of $\lambda$ for $m_{h_{1}}$ and $m_{h_{2}}$ in the left plot of the first row in Fig. 3. We find that for the whole range of $\lambda$ in the plot the impact of the genuine NMSSM corrections of $\mathcal{O}\left(\lambda Y_{t}, \lambda^{2}\right)$ remains less than $0.5 \mathrm{GeV}$. The largest difference between the two approximations occurs for the light singlet-like state $h_{1}$ at large values of $\lambda$ close to the upper limit of $\lambda \approx 0.32$ shown in the plot. In fact, for $m_{h_{1}}$ the difference between the two approximations is seen to rise sharply for increasing values of $\lambda$. On the other hand, at the $\lambda$ value where the cross-over behaviour occurs, $\lambda_{\mathrm{c}}^{(1)}$, the difference between the two approximations is seen to have a local maximum but remains small, below $0.1 \mathrm{GeV}$. For the doublet-like state, which has a one-loop mass of more than $130 \mathrm{GeV}$ (see Fig. 1), the corrections from genuine NMSSM-contributions remain below the level of $1 \%$ over the whole range of $\lambda$. Thus, the approximation based on the MSSM-type contributions is seen to provide a very accurate prediction for the top/stop-sector contributions to the mass of a doublet-like state. For the singlet-like state, where the deviation grows with $\lambda$, the deviation reaches $\approx 1 \%$ for the one-loop mass of the singlet-like state of $\approx 40 \mathrm{GeV}$ for $\lambda \approx 0.32$.

The sharp increase of the corrections of $\mathcal{O}\left(\lambda Y_{t}, \lambda^{2}\right)$ for the highest values of $\lambda$ that is visible for the light singletlike field in the upper left plot of Fig. 3 indicates that the approximation for the stop sector of making the restriction of the MSSM-type contributions becomes questionable for the singlet-like state in this region. However, as shown in the upper right plot of Fig. 3, in this parameter region the stop sector as a whole ceases to provide a reliable approximation of the full one-loop contributions. In the right plot the difference between the full result and the approximation based on the leading MSSM-type contributions from the top/stop sector, $\Delta m_{h_{i}}=\left|m_{h_{i}}^{(1 \mathrm{~L})}-m_{h_{i}}^{t / \tilde{t}-\mathrm{MSSM}}\right|$, is shown together with $\Delta m_{h_{i}}=\left|m_{h_{i}}^{(1 \mathrm{~L})}-m_{h_{i}}^{t / \tilde{t}-\mathrm{MSSM}+\mathrm{HG}}\right|$, where in the latter case the leading MSSM-type contributions from the top/stop sector are supplemented by the contribution from the Higgshiggsino and gauge/gaugino sectors. While for the singletlike state the deviation between the leading contributions from the top/stop sector and the full one-loop result becomes huge for the largest values of $\lambda$, reaching the level of $20 \mathrm{GeV}$, the deviations stay small, far below the level of $1 \mathrm{GeV}$, if the leading contributions from the top/stop sector are supplemented by the contributions from the Higgs-/higgsino and gauge-gaugino sectors. This result for the singlet-like state can be understood from the fact that the gauge couplings of the singlet-like state are heavily suppressed and that therefore the leading contributions for large $\lambda$ arise from the Higgs and higgsino sector. Thus, improving on the approximation of MSSM-type contributions in the stop sector requires the incorporation of the contributions from the Higgs and higgsino sector, while the genuine NMSSM contributions in the stop sector are of minor significance in this context.

For the doublet-like state, namely $h_{1}$ for values $\lambda \lesssim \lambda_{\mathrm{c}}$ and $h_{2}$ for $\lambda \gtrsim \lambda_{\mathrm{c}}$, the difference between the full one-loop result and the result based on the leading contributions from the top/stop sector amounts to a shift of about $5 \mathrm{GeV}$, which is essentially independent of $\lambda$ except for the region where the cross-over behaviour occurs. This nearly constant shift arises mainly from subleading contributions in the top/stop sector. As indicated by the dashed lines, the inclusion of the contributions from the Higgs and the gauge sector reduces the difference from the full one-loop result by about $1 \mathrm{GeV}$.

\subsubsection{Scenario P1}

The difference between the mass predictions in the two approximations in the top/stop sector is plotted as a function of $\lambda$ for $m_{h_{1}}$ and $m_{h_{2}}$ in the left plot of the second row in Fig. 3. As in Fig. 1, the qualitative behaviour is similar to the one in the sample scenario, while the allowed range in $\lambda$ in $\mathrm{P} 1$ is restricted to the region below the cross-over point. The impact of the genuine NMSSM corrections is even smaller in this case than in the sample scenario, amounting to less than $100 \mathrm{MeV}$ for both lighter $\mathcal{C} \mathcal{P}$-even Higgs fields. In the right plot of the second row the difference between the full result and the approximation based on the leading MSSM-type con- 

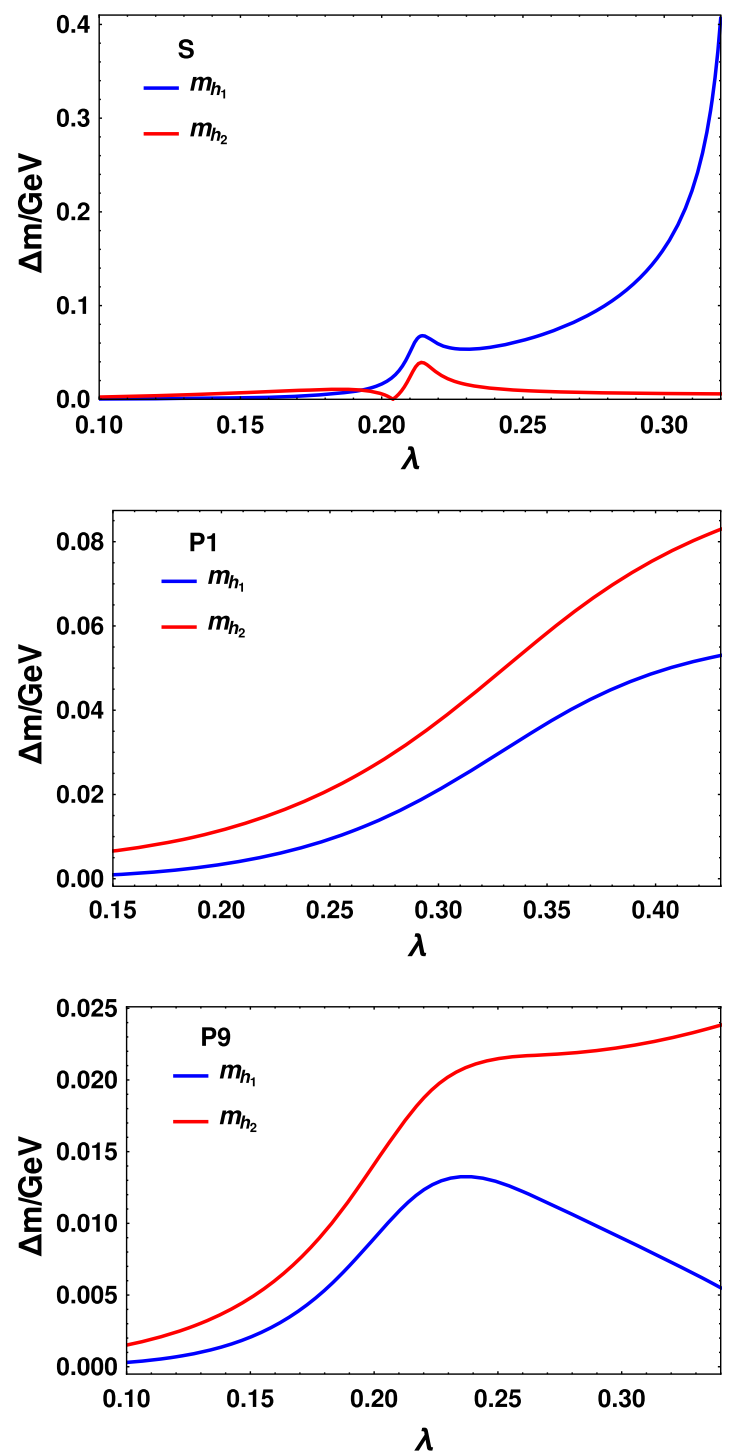

Fig. 3 Absolute difference between partial and full results for the one-loop masses of the two lighter $\mathcal{C P}$-even fields in the sample scenario (first row) and the scenarios P1 (second row) and P9 (third row). Left column absolute difference between the mass predictions including and excluding the genuine NMSSM contributions from the stops of $\mathcal{O}\left(\lambda Y_{t}, \lambda^{2}\right)$ for $m_{h_{1}}$ and $m_{h_{2}}$. Right column absolute differences between the mass predictions based on two different oneloop approximations and the full one-loop result. The solid lines,

tributions from the top/stop sector and the leading MSSMtype contributions from the top/stop sector supplemented by the contribution from the Higgs-higgsino and gauge/gaugino sectors are shown. By supplementing the partial one-loop results with the Higgs/higgsino/gauge-boson/gaugino contributions the mass prediction for the doublet-like state is improved by $\approx 1.5 \mathrm{GeV}$. As explained above for the sample scenario, the difference between the approximate mass prediction and the full one-loop result for the doublet-like
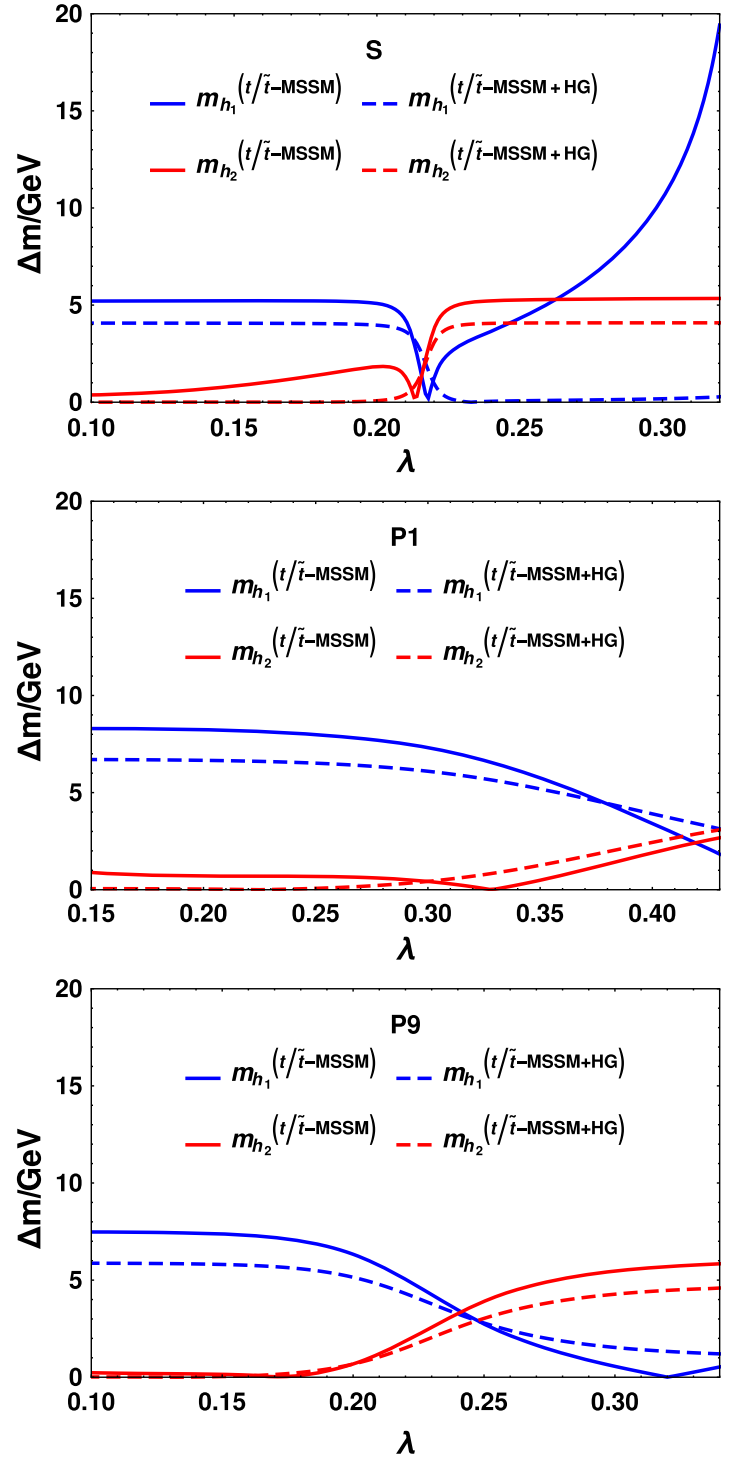

labelled " $t / \tilde{t}$-MSSM", depict the difference between the full result and the approximation based on the leading MSSM-type contributions from the top/stop sector, $\Delta m_{h_{i}}=\left|m_{h_{i}}^{(1 \mathrm{~L})}-m_{h_{i}}^{t / \tilde{t} \text {-MSSM }}\right|$. The dashed lines, labelled " $t / \tilde{t}$-MSSM + HG", show the corresponding result where the leading MSSM-type contributions from the top/stop sector are supplemented by the contributions from the Higgs/higgsino and gauge/gaugino sectors, $\Delta m_{h_{i}}=\left|m_{h_{i}}^{(1 \mathrm{~L})}-m_{h_{i}}^{t / \tilde{t}-\mathrm{MSSM}+\mathrm{HG} \mid}\right|$

state is mainly due to MSSM-type subleading contributions in the top/stop sector. The different variation with $\lambda$ in the right plot as compared to the sample scenario is related to the much wider cross-over region in this case (starting at about $\lambda=0.2$ ). The large deviation encountered in the sample scenario for the singlet-like state above the cross-over region is obviously not present in scenario P1, as the latter one is confined to $\lambda$ values below the cross-over region. 

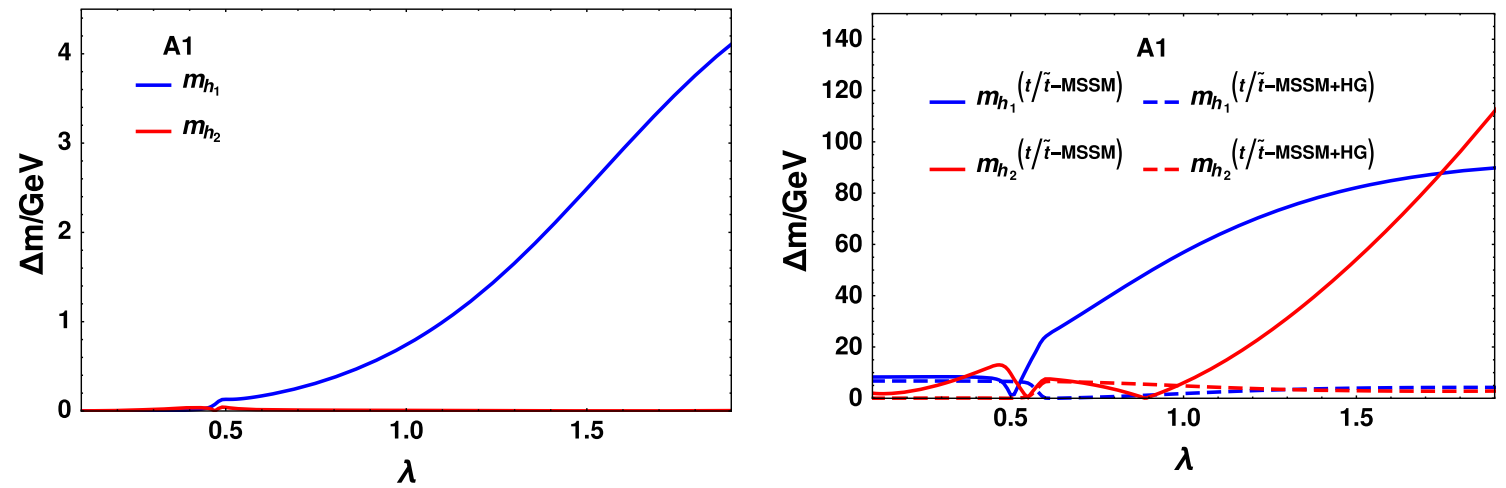

Fig. 4 Absolute difference between partial and full results for the one-loop masses of the two lighter $\mathcal{C} \mathcal{P}$-even fields in the scenario A1. The meaning of the displayed curves is the same as in Fig. 3

\subsubsection{Scenario P9}

For the scenario P9 the results are given in the same fashion as for the other two scenarios in the third row of Fig. 3. As can be seen in the left plot, the impact of the genuine NMSSM corrections is even still smaller than in scenario P1, amounting to less than $25 \mathrm{MeV}$ for both lighter $\mathcal{C P}$ even Higgs fields. By supplementing the partial one-loop results with the Higgs/higgsino/gauge-boson/gaugino contributions (right plot) the mass prediction for the doublet-like state is improved by $\approx 1.5 \mathrm{GeV}$. The overall variation with $\lambda$ resembles the one of the sample scenario (upper right plot) if in the latter case one focuses on $\lambda$ values up to just above the cross-over region. The fact that in the scenario P9 there is a sizeable admixture of singlet- and doublet-components in the states $h_{2}$ and $h_{1}$ above the cross-over region leads to slight modifications. While for the sample scenario $\Delta m$ is the same above and below the cross-over region for the doublet-like state, for scenario P9 we find that $\Delta m$ is somewhat reduced for the doublet-like state above the cross-over region. Thus, in this region the singlet admixture of more than $40 \%$ to $h_{2}$ shifts the approximate one-loop mass prediction closer to the mass obtained with the full one-loop calculation. It should be noted that even in the case of a sizeable admixture of singlet- and doublet-contributions, which is realised in scenario $\mathrm{P} 9$ above the cross-over region, the genuine NMSSM-type contributions have a minor impact compared to the Higgs/higgsino/gauge-boson/gaugino contributions.

\subsubsection{Scenario Al}

For the scenario A1 the corresponding analysis is shown in Fig. 4. As in Figs. 1 and 2, the qualitative behaviour for values close to and below the cross-over region is similar to the sample scenario. Up to values $\lambda \approx 0.7$ the genuine NMSSMtype corrections from the top/stop sector are of similar size as for the sample scenario, amounting up to $\approx 100 \mathrm{MeV}$ for the doublet-like field $h_{1}$ field with a mass close to $125 \mathrm{GeV}$. For the singlet-like field the NMSSM-type contributions from the top/stop sector amount up to $\approx 500 \mathrm{MeV}$ in this region. The NMSSM-type contributions from the top/stop sector increase sharply for the singlet-like field $h_{1}$ at values above $\lambda_{\mathrm{c}}$, amounting up to $4 \mathrm{GeV}$ for the largest values for $\lambda$, and become tiny for the doublet-like field $h_{2}$, staying well below $20 \mathrm{MeV}$.

As before we observe also for this scenario with very large values of $\lambda$ that other contributions beyond the leading MSSM-type contributions from the top/stop sector are numerically much more important than the leading genuine NMSSM-type contributions from the top/stop sector. As can be seen in the right plot of Fig. 4, the difference between the leading MSSM-type contributions from the top/stop sector and the full one-loop result amounts to about $8 \mathrm{GeV}$ for the doublet-like field $h_{1}$ in the region where $\lambda \lesssim 0.5$. Supplementing the leading MSSM-type contributions from the top/stop sector with the Higgs/higgsino/gaugeboson/gaugino contributions improves the prediction by about $1-2 \mathrm{GeV}$. As before, the remaining difference in this parameter region is mainly caused by subleading contributions from the top/stop sector. For the singlet-like state $h_{2}$ the discrepancy between the full one-loop result and the leading MSSM-type contributions from the top/stop sector becomes very significant for increasing $\lambda$, reaching about $12 \mathrm{GeV}$ for $\lambda \approx 0.45$. This large effect is caused by the contributions of the Higgs/higgsino/gauge-boson/gaugino sectors. Incorporating those contributions reduces the discrepancy below the level of $100 \mathrm{MeV}$. For $\lambda \gtrsim 0.5$ the discrepancy between the full one-loop result and the leading MSSM-type contributions from the top/stop sector becomes huge for $h_{1}$. The same is true for $h_{2}$ for very large values of $\lambda$ above 1 . This huge effect is again caused by the contributions of the Higgs/higgsino/gauge-boson/gaugino sectors. Incorporating those contributions reduces the discrepancy to the level of 3-5 GeV. Accordingly, even for this extreme scenario the top/stop sector is well described by just the MSSM-type 
Table 4 Main calculational differences between NMSSMCalc and our result (labelled NMSSMFeynHiggs) in the set-up used for the comparison in Sect. 4.4. The difference at one-loop order is caused only by the different renormalisation of the electric charge, described in
Sect. 2.3. At two-loop order the codes in this set-up only differ by the genuine NMSSM contributions of $\mathcal{O}\left(Y_{t} \lambda \alpha_{s}, \lambda^{2} \alpha_{s}\right)$. The two-loop MSSM corrections beyond $\mathcal{O}\left(\alpha_{t} \alpha_{s}\right)$ and the resummation of logarithms are switched off in NMSSMFeynHiggs for the comparison in Sect. 4.4

\begin{tabular}{llll}
\hline & NMSSMCalc & NMSSM-FeynHiggs \\
\hline One loop & $\alpha_{\mathrm{em}}\left(M_{Z}\right)$ renormalised & $\leftrightarrow$ & $\alpha_{\text {em }}\left(M_{Z}\right)$ reparametrised \\
Two loop & NMSSM $\mathcal{O}\left(\alpha_{t} \alpha_{s}\right)$ & $\leftrightarrow$ & MSSM $\mathcal{O}\left(\alpha_{t} \alpha_{s}\right)$ \\
\hline
\end{tabular}

contributions in those regions of the parameter space where the top/stop sector itself provides an adequate approximation of the full one-loop result. For the highest values of $\lambda$ in this scenario the contributions beyond the top/stop sector are huge, demonstrating the necessity to use in this case a complete result incorporating also the contributions from the Higgs/higgsino and gauge-boson/gaugino sectors.

\subsubsection{Conclusion}

As a result of the comparison performed in this section the MSSM-type top/stop-sector contributions of $\mathcal{O}\left(Y_{t}^{2}\right)$ have been verified as the leading one-loop contributions to MSSMlike fields. The genuine NMSSM top/stop-sector contributions of $\mathcal{O}\left(\lambda Y_{t}, \lambda^{2}\right)$ have the largest impact on singlet-like fields for large values of $\lambda$, where, however, an approximation based only on contributions from the fermion/sfermion sector is in any case insufficient. Our analysis at the oneloop level therefore shows that approximating the result for the top/stop sector by the leading MSSM-type contributions turns out to work well in the parameter regions where the top/stop sector itself yields a reasonable approximation of the full result. These findings provide a strong motivation for applying the same kind of approximation also at the two-loop level. For the description of singlet-like fields in the region of large values of $\lambda$ we have demonstrated the importance of incorporating also the contributions from the Higgs/higgsino and gauge-boson/gaugino sectors.

\subsection{Comparison with NMSSMCalc}

For the comparison of our results with available tools the code NMSSMCalc [65] is particularly suitable, since it is the only public tool using also a mixed $\overline{\mathrm{DR}}$ /on-shell renormalisation scheme. In this section the numerical differences between the results for the masses of the two lighter Higgs states from NMSSMCalc and our calculation will be discussed at different orders for the scenarios given in Table 2 and Table 3. Both codes, NMSSMCalc and our calculation, labelled NMSSM-FeynHiggs in the following, have been adapted for this comparison. The two codes interpret the input parameters in the stop sector as defined for on- shell renormalised masses of the stops. ${ }^{8}$ Since NMSSMCalc uses a different charge renormalisation associated with the value $\alpha\left(M_{Z}\right)$ for the electromagnetic coupling constant, we have reparametrised our result as described in Sect. 2.3. The numerical values for $\alpha\left(M_{Z}\right)$ and $\Delta \alpha$ have been taken directly from NMSSMCalc for this comparison,

$$
\begin{aligned}
& \Delta \alpha=\Delta \alpha_{\text {had }}^{(5)}+\Delta \alpha_{\text {lep }}=5.89188 \cdot 10^{-2}, \\
& \alpha\left(M_{Z}\right)=1 / 128.962 .
\end{aligned}
$$

After the reparametrisation is applied the only difference between the one-loop Higgs-mass predictions of NMSSMFeynHiggs and NMSSMCalc stems from the finite contribution of $\delta v$ used in NMSSMCalc. Beyond the one-loop level only MSSM two-loop contributions of $\mathcal{O}\left(\alpha_{t} \alpha_{s}\right)$ (calculated for on-shell renormalised top and stop masses) are considered in NMSSM-FeynHiggs for this comparison, as only their NMSSM-counterparts are implemented in NMSSMCalc. Two-loop corrections beyond the ones of $\mathcal{O}\left(\alpha_{t} \alpha_{s}\right)$ as well as the resummation of logarithms, which are incorporated in the default version of NMSSM-FeynHiggs, are not included for the analysis in this section (for a discussion of their size see Sect. 4.5). For simplicity, we will refer to this reduced set of two-loop contributions as "two-loop order" throughout this section. The remaining differences between the Higgsmass calculations of NMSSMCalc and NMSSMFeynHiggs in this set-up are summarised in Table 4. The applied modifications ensure that the comparison between the codes will quantify the numerical impact of the genuine NMSSM twoloop corrections of $\mathcal{O}\left(Y_{t} \lambda \alpha_{s}, \lambda^{2} \alpha_{s}\right)$.

We used the SM parameters as specified in the built-in standard input files of NMSSMCalc for this comparison. We passed over the input values in the quark and squark sectors as on-shell parameters from NMSSMFeynHiggs to NMSSM$\mathrm{Calc}$. The pole mass for the top, $m_{t}=173.2 \mathrm{GeV}$, has been used in the loop contributions in this section, and the renormalisation scale has been chosen as $m_{t}$. For the comparison the identical value $\left.\alpha_{s} \overline{\mathrm{MS}}_{(} m_{t}^{(\mathrm{OS})}\right)=0.1069729$ has been used for both codes (using the evaluation in NMSSMCalc with the routines of [88]).

\footnotetext{
8 We thank Kathrin Walz for providing a modified version of NMSSMCalc for this feature.
} 
Table 5 Mass of the lightest $\mathcal{C P}$-even Higgs fields obtained in the MSSM limit with NMSSMCalc and NMSSMFeynHiggs with the reparametrisation to $\alpha\left(M_{Z}\right)$. Both codes yield the identical results in this limit

\begin{tabular}{lllll}
\hline \multicolumn{1}{c}{$\begin{array}{l}\text { Sample } \\
\text { scenario }\end{array}$} & Scenario P1 & Scenario P9 & Scenario A1 \\
\hline$m_{h_{1}}$ MSSM limit & & & \\
GeV & & & \\
Two loop & 116.902 & 109.579 & 115.155 & 109.685 \\
One loop & 140.742 & 115.154 & 152.526 & 151.293 \\
\hline
\end{tabular}

In a first step the one- and two-loop results of NMSSMCalc and NMSSMFeynHiggs have been compared in the MSSM limit, where $\lambda$ and $\kappa$ vanish simultaneously. Both the effects of the different renormalisation schemes and the reparametrisation have to vanish in this limit and thus the results have to be identical. The one- and two-loop results for the mass of the lightest $\mathcal{C P}$-even field obtained in this limit with both codes, given in Table 5, are in agreement with each other with a precision of better than $1 \mathrm{MeV}$ for each scenario (the same holds in this limit also for the predictions for the other neutral Higgs bosons).

This confirms that the MSSM-contributions are treated identically in both calculations. Thus all observed differences between the results for non-vanishing values of $\lambda$ and $\kappa$ can be associated to the treatment of the genuine NMSSMcontributions and residual higher-order effects of the different renormalisation of $v$ after the reparametrisation.

\subsubsection{Sample scenario}

For the sample scenario defined in Table 2 the differences between the two mass predictions are plotted in Fig. 5 as functions of $\lambda$ for the two lighter $\mathcal{C P}$-even states at one- and two-loop order, $\Delta m_{h_{i}}=m_{h_{i}}^{\text {NMSSM-FH }}-m_{h_{i}}^{\text {NMSSMCalc }}$. The left plot in Fig. 5 shows the mass for the lighter state $h_{1}$, and the mass for the heavier state $h_{2}$ is shown in the right plot. The state $h_{1}$ behaves doublet-like for values $\lambda \lesssim \lambda_{c}^{(n)}$ and singlet-like for values $\lambda \gtrsim \lambda_{\mathrm{c}}^{(n)}$ (the behaviour of $h_{2}$ is the opposite), where $\lambda_{\mathrm{c}}^{(1)} \approx 0.21$ and $\lambda_{\mathrm{c}}^{(2)} \approx 0.24 .{ }^{9}$ The values of $\Delta m_{h_{i}}$ are seen to be negative for the doublet-like field and positive for the singlet-like field. We find that the difference between the two results is small for both mass predictions over the whole range of $\lambda$. As expected, the largest differences, reaching about $90 \mathrm{MeV}$ for $\Delta m_{h_{1}}$ at the one-loop level, occur for the mass of the singlet-like state for the largest values of $\lambda$ in the plot. The mass of the doublet-like state is affected to a lesser extent by approximating the $\mathcal{O}\left(\alpha_{t} \alpha_{s}\right)$ cor-

\footnotetext{
$\overline{9}$ These values for $\lambda_{c}^{(n)}$ slightly differ from the ones quoted in Fig. 1 since our result in Fig. 1 has been parametrised in terms of $G_{F}$, while for the comparison with NMSSMCalc we have parametrised the result in terms of $\alpha\left(M_{Z}\right)$.
}

rection by the MSSM-type contributions. The general shape of the one-loop difference, caused by the different treatment of the charge renormalisation, is seen to be maintained at the two-loop level. The main feature at the two-loop level is the shift in the cross-over points by $\Delta \lambda_{\mathrm{c}}=\lambda_{\mathrm{c}}^{(2)}-\lambda_{\mathrm{c}}^{(1)} \approx 0.03$ between two- and one-loop order, while otherwise the difference in the $\mathcal{O}\left(\alpha_{t} \alpha_{s}\right)$ contributions is found to have a very small effect. This can be seen for instance by comparing the local and global extrema at $\lambda_{c}^{(n)}$ and for the largest values around $\lambda_{\max }$. Specifically, for $\lambda=0.32$ we find that the impact of the genuine NMSSM contributions of $\mathcal{O}\left(\alpha_{t} \alpha_{s}\right)$, which are implemented only in NMSSMCalc amounts to less than $50 \mathrm{MeV}$ for $h_{1}$ (for a Higgs mass of $m_{h_{1}} \approx 40 \mathrm{GeV}$ ). For $h_{2}$ the comparison of the height of the local extrema in the cross-over region yields a difference below $20 \mathrm{MeV}$ (for a Higgs mass of $m_{h_{2}} \approx 125 \mathrm{GeV}$ ).

\subsubsection{Scenarios $P 1, P 9$ and $A 1$}

The comparisons between NMSSMFeynHiggs and NMSSM$\mathrm{Calc}$ for the scenarios P1 and P9 are shown in Fig. 6 in the first and second row, while the scenario A1 is shown in the lower row. For better illustration we plot here the size of the two-loop contributions, $\Delta m_{h_{i}}=m_{h_{i}}^{1 \mathrm{~L}}-m_{h_{i}}^{2 \mathrm{~L}}$, as obtained with the two codes as a function of $\lambda$. As for the sample scenario, the main effect in the comparison arises from a slight relative shift in $\lambda$ between the predictions of the two codes. At the one-loop level this shift amounts typically to $\Delta \lambda_{\mathrm{c}} \approx 10^{-4}$ (the corresponding plots are not shown here since the curves for the predictions of the two codes would be essentially indistinguishable). For the two-loop contributions displayed in Fig. 6 one can see that the genuine NMSSM-type two-loop corrections that are implemented in NMSSMCalc give rise to a slightly different dependence on $\lambda$, which becomes visible for large values of $\lambda$.

As discussed above, in the P1 scenario the displayed range of $\lambda$ corresponds to the region below the cross-over point. For the sample scenario we found in this region a slight increase in the absolute difference between the results; see Fig. 5. The difference between the two-loop contributions shown in Fig. 6 is seen to follow a similar pattern. For $h_{1}$ (upper left plot) the difference between the two contributions exceeds the level of $0.5 \mathrm{GeV}$ for the highest values of $\lambda$ that are possible in this scenario because of the steep slope of the curves (which are slightly shifted in $\lambda$ with respect to each other) in this region. The dominantly doublet-like state $h_{1}$ has a significant singlet admixture in this region, which increases up to more than $30 \%$ for the highest $\lambda$ values. It should be noted that such a large singlet admixture severely worsens the compatibility of the state $h_{1}$ with the observed Higgs signal at about $125 \mathrm{GeV}$ (independently of its mass, which is incompatible with the signal in this part of the plot; see Fig. 1). The differences are smaller for the (dominantly singlet-like) state 


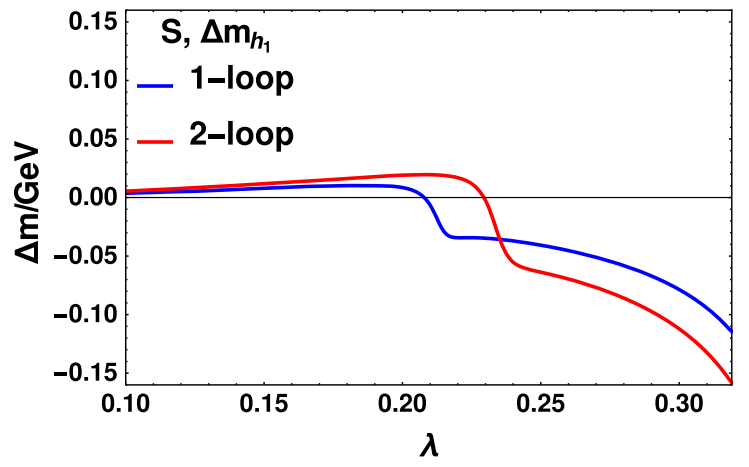

Fig. 5 Difference between the mass predictions for the two lighter $\mathcal{C P}$ even fields $h_{1}$ and $h_{2}$ from NMSSMCalc and NMSSM-FeynHiggs at one- and two-loop order, $\Delta m_{h_{i}}=m_{h_{i}}^{\mathrm{NMSS}-\mathrm{FH}}-m_{h_{i}}^{\mathrm{NMSSMCalc}}$, for the sample scenario. The result of NMSSM-FeynHiggs has been

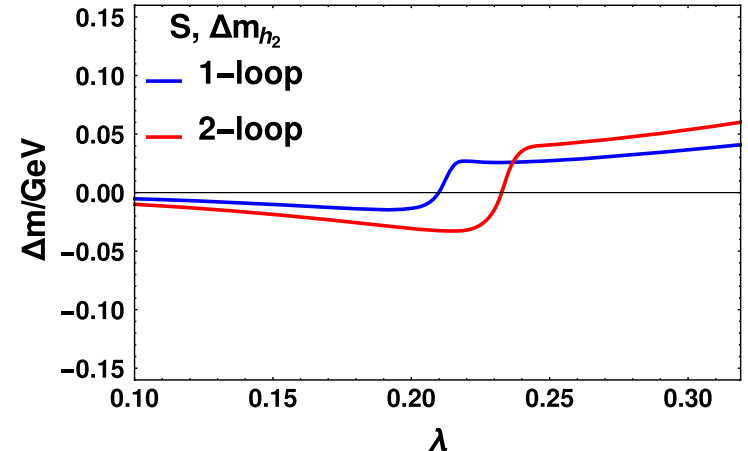

reparametrised to $\alpha\left(M_{Z}\right)$. The points where the cross-over behaviour of the fields $h_{1}$ and $h_{2}$ occurs at one- and two-loop order are $\lambda_{\mathrm{c}}^{(1)} \approx 0.21$ and $\lambda_{\mathrm{c}}^{(2)} \approx 0.24$
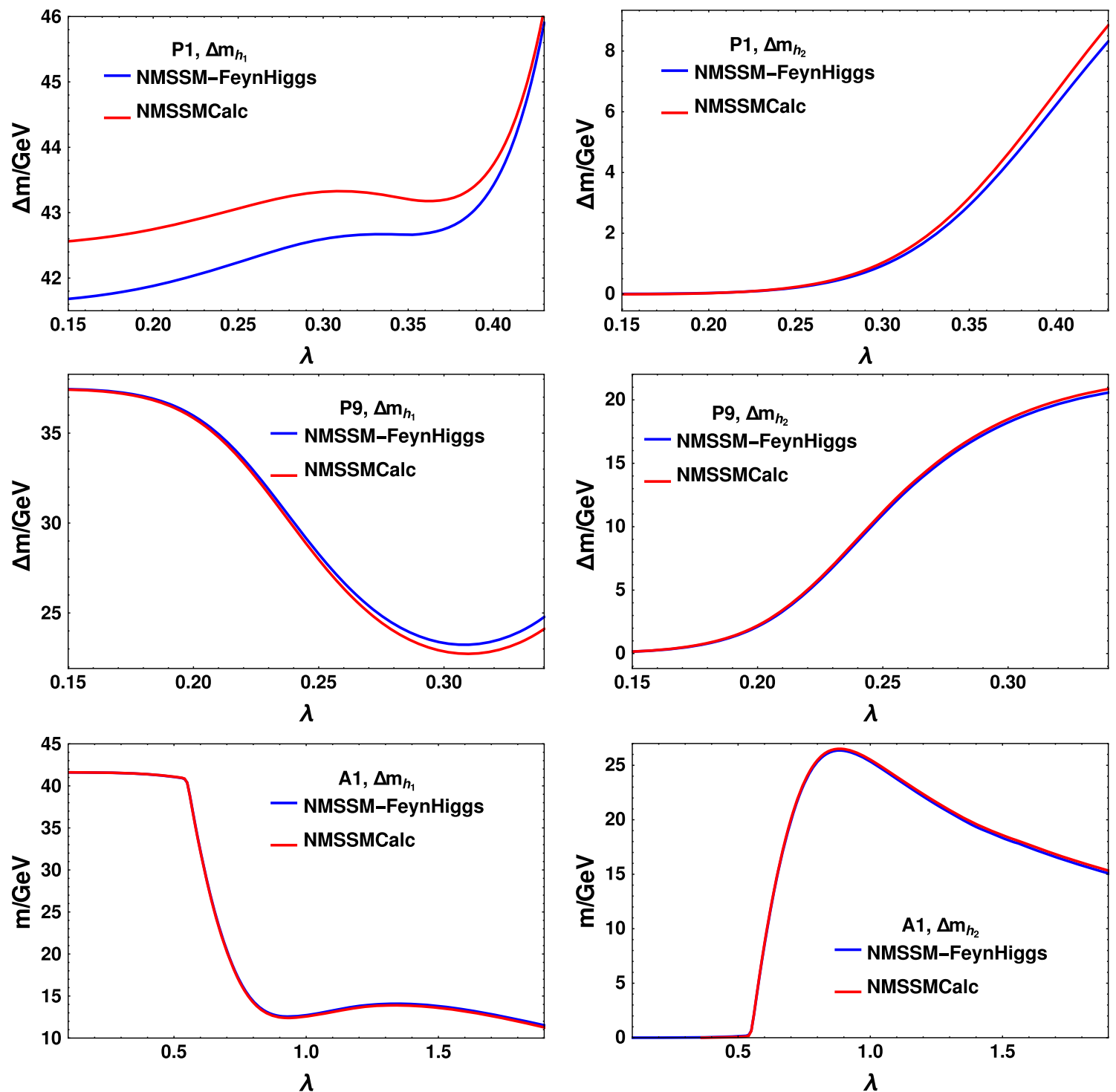

Fig. 6 Size of the two-loop contributions, $\Delta m_{h_{i}}=m_{h_{i}}^{1 \mathrm{~L}}-m_{h_{i}}^{2 \mathrm{~L}}$ evaluated with NMSSMFeynHiggs (blue) and with NMSSMCalc (red) for the masses of the two lighter $\mathcal{C P}$-even fields $h_{1}$ (left) and $h_{2}$ (right) in the scenarios P1 (upper row), P9 (second row) and A1 (third row) 
$h_{2}$ (upper right plot) and reach a significant level only for $\lambda$ values that are close to the boundary of the allowed range.

For the scenario P9, where above the cross-over region a relatively large admixture of more than $40 \%$ between the doublet-like and the singlet-like state occurs, the differences stay relatively small over the whole displayed range of $\lambda$ both for $h_{1}$ (middle left plot) and $h_{2}$ (middle right plot). The largest deviations occur for the dominantly singlet-like state $h_{1}$ (with a sizeable doublet admixture) for the highest values of $\lambda$ above the cross-over region, where the two-loop contributions differ from each other by up to $0.8 \mathrm{GeV}$.

For the scenario A1, where above the cross-over region a relatively large admixture of more than $30 \%$ between the doublet-like and the singlet-like state occurs, the differences nevertheless stay small over the whole displayed range of $\lambda$ both for $h_{1}$ (lower left plot) and $h_{2}$ (lower right plot). Even for the largest values of $\lambda$ the difference between the two contributions remains below $0.26 \mathrm{GeV}$. Our analysis shows that even for this extreme scenario with very high values of $\lambda$ the genuine NMSSM-type two-loop corrections that are only implemented in NMSSMCal $\mathrm{C}$ are of minor numerical significance. From our analysis at the one-loop level, on the other hand, it is expected that the two-loop contributions beyond the fermion/sfermion sector are very important in this parameter region, so that the theoretical uncertainties of both codes are expected to be rather large in this region.

\subsubsection{Conclusion}

The results shown in Figs. 5 and 6 confirm that the approximation in terms of MSSM-type contributions at the twoloop level induces an uncertainty that is numerically small, if $\lambda<Y_{t}$, as discussed in the previous sections. As expected, the approximation works best for the MSSM-like (doubletlike) fields, and we have found for the analysed scenarios that the deviations stay below the level of $1 \mathrm{GeV}$ even for the highest possible values of $\lambda$ and in regions with a large admixture between double- and singlet-like states. An improved prediction for singlet-like states for large values of $\lambda$ would require the incorporation of two-loop contributions from the Higgs/higgsino and gauge-boson/gaugino sectors, which is beyond the scope of our present analysis.

\subsection{Impact of additional corrections beyond $\mathcal{O}\left(\alpha_{t} \alpha_{S}\right)$}

While the genuine NMSSM two-loop corrections of $\mathcal{O}\left(Y_{t} \lambda \alpha_{S}\right.$, $\left.\lambda^{2} \alpha_{S}\right)$ induce small effects, as discussed in the previous section, the MSSM two-loop corrections beyond $\mathcal{O}\left(\alpha_{t} \alpha_{s}\right)$ and the resummation of large logarithms can result in a shift for the mass of the light doublet-like field of several $\mathrm{GeV}$. In order to quantify the impact of the additional MSSMcontributions of $\mathcal{O}\left(\alpha_{t}^{2}, \alpha_{b} \alpha_{s}, \alpha_{t} \alpha_{b}\right)$ and the resummation of logarithms, which are incorporated in NMSSMFeynHiggs, the results with and without these corrections are plotted as functions of $\lambda$ in Figs. 7 and 8 for the discussed scenarios. Here the one-loop $\overline{\mathrm{MS}}$-value of the top-quark, $m_{t}^{\overline{\mathrm{MS}}}\left(m_{t}\right)$, is used in the loop contributions. A sizeable shift of about $3-$ $8 \mathrm{GeV}$ can be observed for the mass of the doublet-like field. As expected, the impact of the MSSM-type two-loop contributions on the mass prediction for the singlet-like field remains small. In comparison with the contributions discussed in the previous section we find that the effect of the additional corrections beyond $\mathcal{O}\left(\alpha_{t} \alpha_{s}\right)$ can exceed the numerical impact of the genuine NMSSM-corrections of $\mathcal{O}\left(Y_{t} \lambda \alpha_{s}, \lambda^{2} \alpha_{s}\right)$ by more than one order of magnitude.

\section{Conclusions}

We have presented predictions for the Higgs-boson masses in the NMSSM obtained within the Feynman-diagrammatic approach. They are based on the full NMSSM one-loop corrections supplemented with the dominant and sub-dominant two-loop corrections of MSSM-type, including contributions at $\mathcal{O}\left(\alpha_{t} \alpha_{s}, \alpha_{b} \alpha_{s}, \alpha_{t}^{2}, \alpha_{t} \alpha_{b}\right)$, as well as a resummation of leading and subleading logarithms from the top/scalar top sector. In order to enable a direct comparison with the corresponding results in the MSSM, the renormalisation scheme and all parameters and conventions have been chosen such that the well-known MSSM result of the code FeynHiggs is recovered in the MSSM limit of the NMSSM.

In our phenomenological analysis we have first investigated a scenario where depending on the value of $\lambda$ either the lightest or the next-to-lightest neutral Higgs state can be identified with a SM-like Higgs boson at about $125 \mathrm{GeV}$. Furthermore we have investigated two scenarios (originally proposed in a different context; see the discussion in Sect. 4.1) where larger values of $\lambda$ than in the sample scenario can be realised, and sizeable admixtures between singlet- and doublet-like states can occur also outside of the "cross-over" region. The lightest neutral Higgs-state can be identified with a SM-like Higgs boson at about $125 \mathrm{GeV}$ in both scenarios for low and moderate values of $\lambda$. As expected, the state that can be identified with the observed Higgs signal at about $125 \mathrm{GeV}$ is doublet-like in all cases, i.e. it receives only relatively small contributions from the singlet state of the NMSSM. In order to investigate the impact of the various contributions for even higher values of $\lambda$, we have furthermore analysed another variation of these scenarios in which values of $\lambda$ up to $\lesssim 1.5$ can be realised. The inclusion of the higher-order contributions which are known for the MSSM is crucial for all scenarios in order to obtain an accurate prediction for the mass spectrum.

We have investigated different approximations at the oneloop level in comparison with our full one-loop result for the 

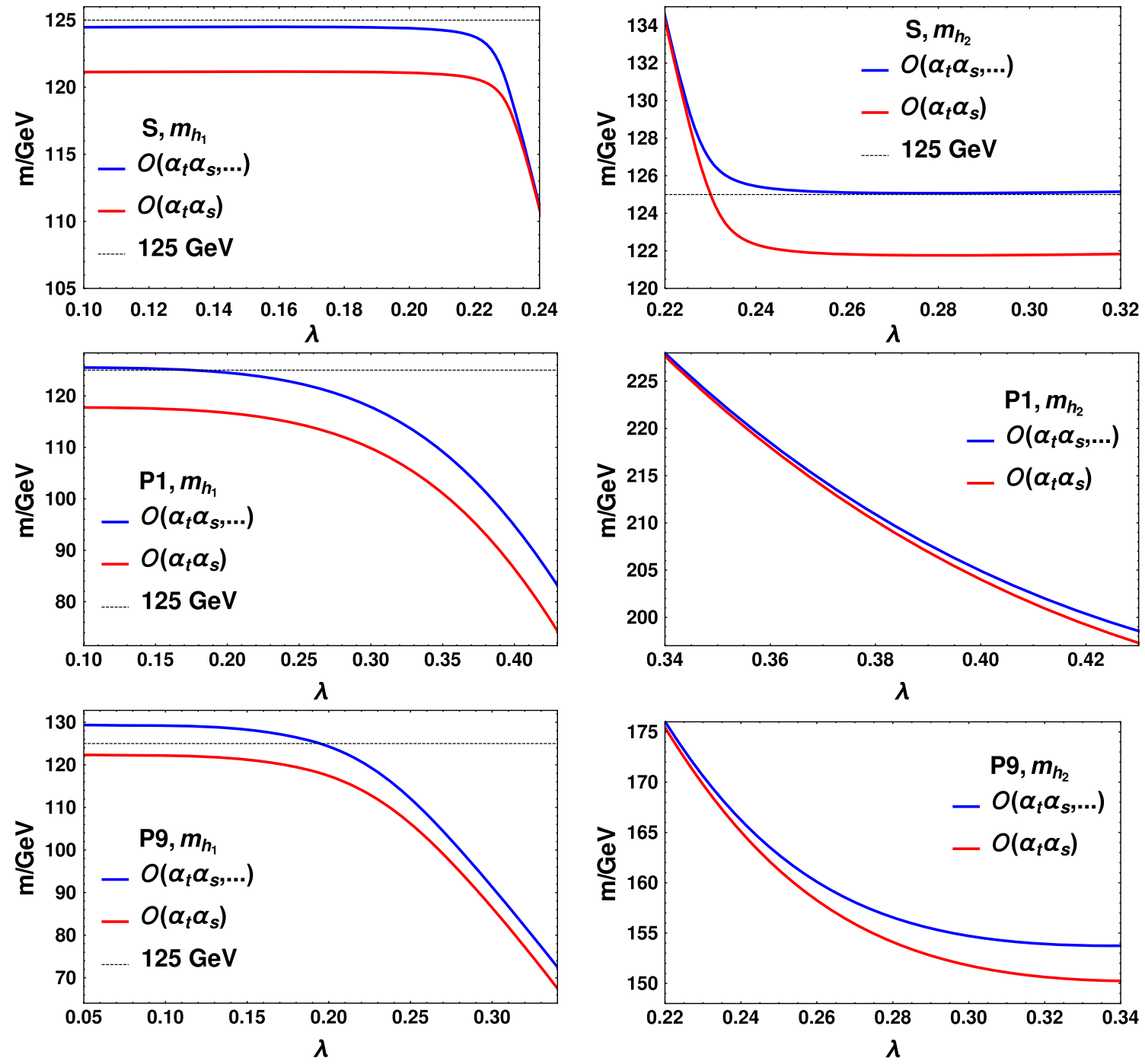

Fig. 7 Mass predictions for the two lighter $\mathcal{C} \mathcal{P}$-even fields $h_{1}$ and $h_{2}$ for different contributions at two-loop order in the sample scenario (first row) and the scenarios $\mathrm{P} 1$ (second row) and $\mathrm{P} 9$ (third row). The blue lines include all MSSM-type corrections of $\mathcal{O}\left(\alpha_{t} \alpha_{s}, \alpha_{b} \alpha_{s}, \alpha_{t}^{2}, \alpha_{t} \alpha_{b}\right)$

and the resummation of large logarithms as included in FeynHiggs 2.10.2, while for the red curves only the MSSM-type corrections of $\mathcal{O}\left(\alpha_{t} \alpha_{s}\right)$ are included beyond the full one-loop contributions. The thin horizontal line marks $125 \mathrm{GeV}$
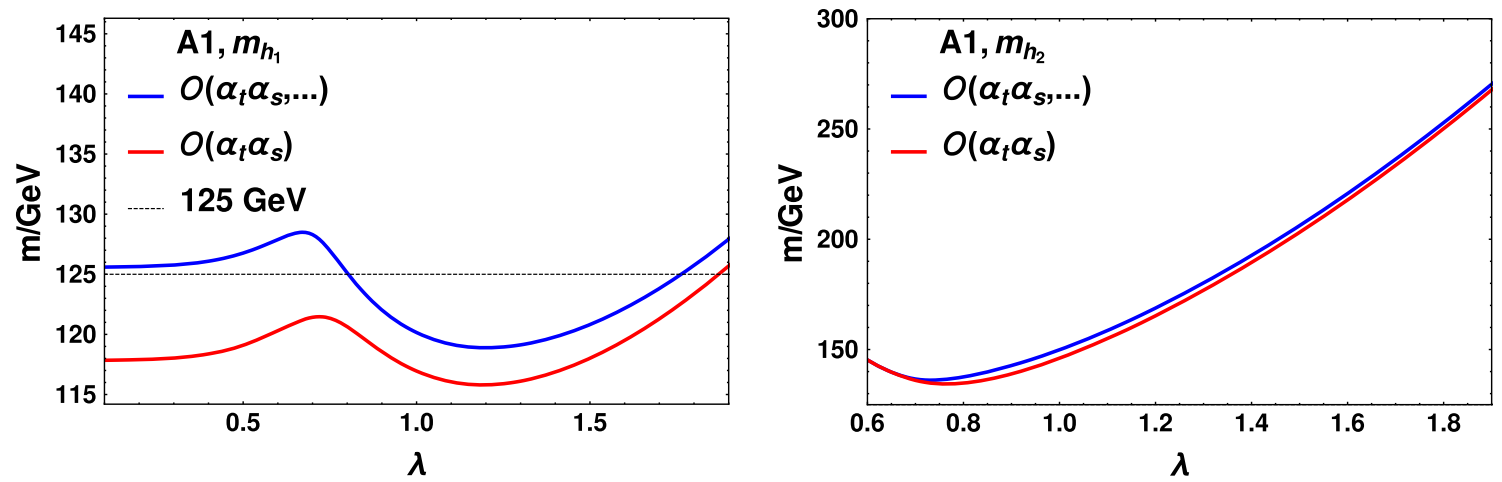

Fig. 8 Mass predictions for the two lighter $\mathcal{C} \mathcal{P}$-even fields $h_{1}$ and $h_{2}$ for different contributions at two-loop order in the scenario A1. The meaning of the displayed curves is the same as in Fig. 7 
NMSSM. We have found that the approximation of the result for the top/stop sector in terms of the leading MSSM-type contributions works well in the parameter regions where the top/stop sector itself yields a reasonable approximation of the full result. It therefore appears to be well motivated to make use of this approximation at the two-loop level. The genuine NMSSM top/stop-sector contributions of $\mathcal{O}\left(Y_{t} \lambda, \lambda^{2}\right)$ can be significant for singlet-like fields if $\lambda$ is large. For such large values of $\lambda$, however, the improvement achieved by including those genuine NMSSM contributions from the top/stop sector is by far overshadowed by the fact that contributions from the Higgs and higgsino sector become more and more important for a singlet-like Higgs field.

We have compared our predictions with the public code NMSSMCalc for on-shell parameters in the top/stop sector. For the purpose of this comparison we have done an appropriate reparametrisation of the electromagnetic coupling constant, and we have switched off the two-loop corrections beyond the ones of $\mathcal{O}\left(\alpha_{t} \alpha_{s}\right)$ as well as the resummation of leading and subleading logarithms in our code. After those adaptations the predictions of the two codes only differ in the charge renormalisation at the one-loop level and in the genuine NMSSM top/stop-sector contributions of $\mathcal{O}\left(Y_{t} \lambda \alpha_{s}, \lambda^{2} \alpha_{s}\right)$ at the two-loop level. Since these differences arise only from contributions beyond the MSSM, agreement between the predictions of the two codes is expected in the MSSM limit of the NMSSM. We have indeed found that the results obtained with the two codes perfectly agree with each other in this case. For the case of the NMSSM we have compared the predictions of the two codes as a function of $\lambda$. We have found that the differences stay small over the whole range of $\lambda$, with a maximum absolute difference in the mass of the singlet- or the doublet-like state below $1 \mathrm{GeV}$ in the considered scenarios. The difference is mainly caused by the different treatment of the charge renormalisation at the oneloop level, while the effect of the genuine NMSSM top/stopsector contributions of $\mathcal{O}\left(Y_{t} \lambda \alpha_{s}, \lambda^{2} \alpha_{s}\right)$ is found to be generally smaller except for the highest values of $\lambda$ that can be realised in the scenarios. The impact of the genuine NMSSM top/stop-sector contributions of $\mathcal{O}\left(Y_{t} \lambda \alpha_{s}, \lambda^{2} \alpha_{s}\right)$ turned out to be small even in parameter regions where the dominantly doublet-like state has a singlet admixture of more than $30 \%$. A more detailed comparison between the two codes will be presented in a forthcoming publication.

As a final step of our numerical analysis we have investigated the impact of the MSSM-corrections beyond $\mathcal{O}\left(\alpha_{t} \alpha_{s}\right)$ and the resummation of large logarithms that are incorporated in our code but not in NMSSMCalc. While those corrections are small for the mass of a dominantly singlet-like state, they amount to an effect of $3-8 \mathrm{GeV}$ for the mass of the doublet-like state in the considered scenarios. This is typically more than an order of magnitude larger than the corresponding effect of the genuine NMSSM-corrections of $\mathcal{O}\left(Y_{t} \lambda \alpha_{s}, \lambda^{2} \alpha_{s}\right)$.

The results presented in this paper will be used as a basis for the extension of the code FeynHiggs to the NMSSM. Our analysis has revealed that for singlet-like states in the parameter region of very high values of $\lambda$ two-loop corrections beyond the fermion/sfermion sector are expected to be sizeable. In order to reduce the theoretical uncertainties in this parameter region the incorporation of two-loop contributions from the Higgs/higgsino and gauge-boson/gaugino sectors will be desirable. Partial results of this kind have only been obtained in a pure $\overline{\mathrm{DR}}$ scheme up to now. We leave a more detailed discussion of this issue to future work.

Acknowledgements We want to thank Ramona Gröber, Margarete Mühlleitner, Heidi Rzehak, Oscar Stål and Kathrin Waltz for interesting discussions, useful input and interfaces with their codes. We thank Melina Gomez Bock, Rachid Benbrik and Pietro Slavich for helpful communication. The work of S.H. is supported in part by CICYT (grant FPA 2013-40715-P) and by the Spanish MICINN's ConsoliderIngenio 2010 Program under grant MultiDark CSD2009-00064. The authors acknowledge support by the DFG through the SFB 676 "Particles, Strings and the Early Universe". This research was supported in part by the European Commission through the "HiggsTools" Initial Training Network PITN-GA-2012-316704.

Open Access This article is distributed under the terms of the Creative Commons Attribution 4.0 International License (http://creativecomm ons.org/licenses/by/4.0/), which permits unrestricted use, distribution, and reproduction in any medium, provided you give appropriate credit to the original author(s) and the source, provide a link to the Creative Commons license, and indicate if changes were made.

Funded by $\mathrm{SCOAP}^{3}$.

\section{References}

1. ATLAS Collaboration, G. Aad et al., Phys. Lett. B 716, 1 (2012). arXiv: 1207.7214

2. CMS Collaboration, S. Chatrchyan et al., Phys. Lett. B 716, 30 (2012). arXiv:1207.7235

3. K. Tackmann, Talk given at LHCP 2015, September 2015, St. Petersburg, Russia, see: https://indico.cern.ch/event/389531/ session/31/contribution/50/attachments/1147367/1645479/ LHCP2015.pdf. Accessed 12 Jan 2017

4. M. Pieri, Talk given at LHCP 2015, September 2015, St. Petersburg, Russia, see: https://indico.cern.ch/event/389531/session/ 31/contribution/51/attachments/1147368/1650410/LHCHCP

MarcoPieri_fin_1.pdf. Accessed 12 Jan 2017

5. H.P. Nilles, Phys. Rep. 110, 1 (1984)

6. H.E. Haber, G.L. Kane, Phys. Rep. 117, 75 (1985)

7. S. Heinemeyer, O. Stal, G. Weiglein, Phys. Lett. B 710, 201 (2012). arXiv: 1112.3026

8. K. Jakobs, G. Quast, G. Weiglein, Higgs-boson physics at the LHC, in The Large Hadron Collider: Harvest of Run 1, ed. by T. SchörnerSadenius (Springer, Berlin, 2015), pp. 195-258

9. U. Ellwanger, C. Hugonie, A.M. Teixeira, Phys. Rep. 496, 1 (2010). arXiv:0910.1785

10. M. Maniatis, Int. J. Mod. Phys. A 25, 3505 (2010). arXiv:0906.0777

11. J.R. Ellis et al., Phys. Rev. D 39, 844 (1989) 
12. D. Miller, R. Nevzorov, P. Zerwas, Nucl. Phys. B 681, 3 (2004). arXiv:hep-ph/0304049

13. S.F. King, M. Muhlleitner, R. Nevzorov, Nucl. Phys. B 860, 207 (2012). arXiv:1201.2671

14. F. Domingo, G. Weiglein, JHEP 04, 095 (2016). doi:10.1007/ JHEP04(2016)095. arXiv:1509.07283

15. G. Aad, et al., Phys. Rev. Lett. 114, 191803 (2015). doi:10.1103/ PhysRevLett.114.191803. arXiv:1503.07589

16. P.Z. Skands, D. Wicke, Eur. Phys. J. C 52, 133 (2007). arXiv:hep-ph/0703081

17. A.H. Hoang, I.W. Stewart, Nucl. Phys. Proc. Suppl. 185, 220 (2008). arXiv:0808.0222

18. A.H. Hoang, in TOP2014, Cannes, France, September 28-October 3, 2014 (2014). arXiv: 1412.3649

19. S. Heinemeyer, W. Hollik, G. Weiglein, Phys. Rev. D 58, 091701 (1998). arXiv:hep-ph/9803277

20. S. Heinemeyer, W. Hollik, G. Weiglein, Phys. Lett. B 440, 296 (1998). arXiv:hep-ph/9807423

21. S. Heinemeyer, W. Hollik, G. Weiglein, Eur. Phys. J. C 9, 343 (1999). arXiv:hep-ph/9812472

22. R.J. Zhang, Phys. Lett. B 447, 89 (1999). arXiv:hep-ph/9808299

23. J.R. Espinosa, R.J. Zhang, JHEP 03, 026 (2000). arXiv:hep-ph/9912236

24. G. Degrassi, P. Slavich, F. Zwirner, Nucl. Phys. B 611, 403 (2001). arXiv:hep-ph/0105096

25. J.R. Espinosa, R.J. Zhang, Nucl. Phys. B 586, 3 (2000). arXiv:hep-ph/0003246

26. A. Brignole et al., Nucl. Phys. B 631, 195 (2002). arXiv:hep-ph/0112177

27. A. Brignole et al., Nucl. Phys. B 643, 79 (2002). arXiv:hep-ph/0206101

28. S. Heinemeyer et al., Eur. Phys. J. C 39, 465 (2005). arXiv:hep-ph/0411114

29. T. Hahn et al., Phys. Rev. Lett. 112, 141801 (2014). arXiv: 1312.4937

30. P. Draper, G. Lee, C.E.M. Wagner, Phys. Rev. D 89, 055023 (2014). arXiv: 1312.5743

31. G. Lee, C.E.M. Wagner, Phys. Rev. D 92(7), 075032 (2015). doi:10. 1103/PhysRevD.92.075032. arXiv: 1508.00576

32. R. Hempfling, Phys. Rev. D 49, 6168 (1994)

33. L.J. Hall, R. Rattazzi, U. Sarid, Phys. Rev. D 50, 7048 (1994). arXiv:hep-ph/9306309

34. M. Carena et al., Nucl. Phys. B 426, 269 (1994). arXiv:hep-ph/9402253

35. M. Carena et al., Nucl. Phys. B 577, 88 (2000). arXiv:hep-ph/9912516

36. D. Noth, M. Spira, Phys. Rev. Lett. 101, 181801 (2008). arXiv:0808.0087

37. D. Noth, M. Spira, JHEP 06, 084 (2011). arXiv:1001.1935

38. S. Borowka et al., Eur. Phys. J. C 74, 2994 (2014). arXiv: 1404.7074

39. S. Borowka et al., Eur. Phys. J. C 75, 424 (2015). arXiv: 1505.03133

40. G. Degrassi, S. Di Vita, P. Slavich, Eur. Phys. J. C 75, 61 (2015). arXiv: 1410.3432

41. S. Heinemeyer, W. Hollik, G. Weiglein, Comput. Phys. Commun. 124, 76 (2000). arXiv:hep-ph/9812320

42. T. Hahn et al., Comput. Phys. Commun. 180, 1426 (2009)

43. G. Degrassi et al., Eur. Phys. J. C 28, 133 (2003). arXiv:hep-ph/0212020]

44. M. Frank et al., JHEP 0702, 047 (2007). arXiv:hep-ph/0611326

45. FeynHiggs. See: http://www.feynhiggs.de. Accessed 12 Jan 2017

46. S.P. Martin, Phys. Rev. D 71, 116004 (2005). arXiv:hep-ph/0502168

47. S.P. Martin, Phys. Rev. D 75, 055005 (2007). arXiv:hep-ph/0701051

48. R. Harlander et al., Phys. Rev. Lett. 100, 191602 (2008). arXiv:0803.0672
49. P. Kant et al., JHEP 1008, 104 (2010). arXiv:1005.5709

50. S. Heinemeyer, W. Hollik, G. Weiglein, Phys. Rep. 425, 265 (2006). arXiv:hep-ph/0412214

51. O. Buchmueller et al., Eur. Phys. J. C 74, 2809 (2014). arXiv: 1312.5233

52. G. Degrassi, P. Slavich, Nucl. Phys. B 825, 119 (2010). arXiv:0907.4682

53. F. Staub, W. Porod, B. Herrmann, JHEP 10, 040 (2010). arXiv: 1007.4049

54. K. Ender et al., Phys. Rev. D 85, 075024 (2012). arXiv: 1111.4952

55. T. Graf et al., JHEP 10, 122 (2012). arXiv: 1206.6806

56. W. Porod, Comput. Phys. Commun. 153, 275 (2003). arXiv:hep-ph/0301101

57. W. Porod, F. Staub, Comput. Phys. Commun. 183, 2458 (2012). arXiv: 1104.1573

58. M.D. Goodsell, K. Nickel, F. Staub, Phys. Rev. D 91, 035021 (2015). arXiv:1411.4665

59. P. Athron et al., Comput. Phys. Commun. 190, 139 (2015) arXiv: 1406.2319

60. U. Ellwanger, C. Hugonie, Comput. Phys. Commun. 177, 399 (2007). arXiv:hep-ph/0612134

61. F. Domingo, JHEP 06, 052 (2015). doi:10.1007/ JHEP06(2015)052. arXiv:1503.07087

62. B.C. Allanach, Comput. Phys. Commun. 143, 305 (2002). arXiv:hep-ph/0104145

63. B.C. Allanach et al., Comput. Phys. Commun. 185, 2322 (2014). arXiv: 1311.7659

64. B.C. Allanach, A. Bednyakov, R. Ruiz de Austri, Comput. Phys. Commun. 189, 192 (2015). arXiv: 1407.6130

65. J. Baglio et al., Comput. Phys. Commun. 185, 3372 (2014). arXiv: 1312.4788

66. M. Muhlleitner et al., JHEP 05, 128 (2015). doi:10.1007/ JHEP05(2015)128. arXiv:1412.0918

67. F. Staub, P. Athron, U. Ellwanger, R. Gröber, M. Mühlleitner, P. Slavich, A. Voigt, Comput. Phys. Commun. 202, 113 (2016). doi:10.1016/j.cpc.2016.01.005. arXiv:1507.05093

68. W. Hollik, S. Paßehr, Phys. Lett. B 733, 144 (2014). arXiv: 1401.8275

69. O. Stål, G. Weiglein, L. Zeune, JHEP 09, 158 (2015). arXiv: 1506.07465

70. F. Domingo, T. Lenz, JHEP 07, 101 (2011). arXiv:1101.4758

71. J. Kublbeck, M. Bohm, A. Denner, Comput. Phys. Commun. 60, 165 (1990)

72. T. Hahn, Comput. Phys. Commun. 140, 418 (2001). arXiv:hep-ph/0012260

73. T. Hahn, M. Perez-Victoria, Comput. Phys. Commun. 118, 153 (1999). arXiv:hep-ph/9807565

74. T. Riemann (ed.), PoS. LL2012 (2012)

75. F. Staub, Comput. Phys. Commun. 185, 1773 (2014). arXiv: 1309.7223

76. F. Staub, Comput. Phys. Commun. 184, 1792 (2013). arXiv: 1207.0906

77. F. Staub, Comput. Phys. Commun. 182, 808 (2011). arXiv: 1002.0840

78. F. Staub, Comput. Phys. Commun. 181, 1077 (2010). arXiv:0909.2863

79. F. Domingo et al., Eur. Phys. J. C 76, 249 (2016). arXiv: 1602.07691

80. ATLAS Collaboration, ATLAS-CONF-2015-081 (2015)

81. CMS Collaboration, CMS-PAS-EXO-15-004 (2015)

82. P. Bechtle et al., Comput. Phys. Commun. 181, 138 (2010). arXiv:0811.4169

83. P. Bechtle et al., Comput. Phys. Commun. 182, 2605 (2011). arXiv: 1102.1898

84. P. Bechtle et al., Eur. Phys. J. C 74, 2693 (2014). arXiv:1311.0055

85. P. Bechtle et al., PoS CHARGED2012, 024 (2012). arXiv:1301.2345 
86. P. Bechtle et al., Eur. Phys. J. C 75, 421 (2015). arXiv:1507.06706

87. K.E. Williams, H. Rzehak, G. Weiglein, Eur. Phys. J. C 71, 1669 (2011). arXiv:1103.1335
88. K.G. Chetyrkin, J.H. Kuhn, M. Steinhauser, Comput. Phys. Commun. 133, 43 (2000). arXiv:hep-ph/0004189 